\title{
Codimension-2 Bifurcation Analysis and Control of a Discrete Mosquito Model with a Proportional Release Rate of Sterile Mosquitoes
}

\author{
Qiaoling Chen $\mathbb{D}^{1,},{ }^{1,2}$ Zhidong Teng $\mathbb{D},{ }^{3}$ Junli Liu $\mathbb{D}^{1},{ }^{1}$ and Feng Wang ${ }^{4}$ \\ ${ }^{1}$ School of Science, Xi'an Polytechnic University, Xi'an 710048, China \\ ${ }^{2}$ School of Mathematics and Information Science, Shaanxi Normal University, Xi'an 710062, China \\ ${ }^{3}$ College of Mathematics and System Sciences, Xinjiang University, Urumqi 830046, China \\ ${ }^{4}$ School of Mathematics and Statistics, Xidian University, Xi'an 710071, China \\ Correspondence should be addressed to Qiaoling Chen; chenqiaoling@xpu.edu.cn
}

Received 26 March 2020; Accepted 27 July 2020; Published 17 August 2020

Academic Editor: Marcelo Messias

Copyright ( 2020 Qiaoling Chen et al. This is an open access article distributed under the Creative Commons Attribution License, which permits unrestricted use, distribution, and reproduction in any medium, provided the original work is properly cited.

This paper concerns a discrete wild and sterile mosquito model with a proportional release rate of sterile mosquitoes. It is shown that the discrete model undergoes codimension-2 bifurcations with $1: 2,1: 3$, and $1: 4$ strong resonances by applying the bifurcation theory. Some numerical simulations, including codimension-2 bifurcation diagrams, maximum Lyapunov exponents diagrams, and phase portraits, are also presented to illustrate the validity of theoretical results and display the complex dynamical behaviors. Moreover, two control strategies are applied to the model.

\section{Introduction}

In recent years, the mosquito-borne diseases, including West Nile virus, dengue, malaria, Japanese encephalitis, and chikungunya, have attracted much attention. Until now, there are no available vaccines for many of these viruses, which constitute a significant threat to global health. To control the transmissions of mosquito-borne diseases, it is necessary to adopt some strategies to reduce the number of wild mosquitoes. One of effective and environment-friendly approaches is sterile insect technique (SIT). Generally, the mass male mosquitoes are exposed to radiation and thereby rendered sexually sterile. After being released into the desired area, the treated male mosquitoes will mate with wide female mosquitoes and their offspring is nonviable. In this process, choosing the number of sterile mosquitoes and suitable releasing strategy are particularly important.

To understand the interactive dynamics of wide mosquitoes and sterile mosquitoes, some mathematical models have been introduced, for example, [1-9]. Recently, Cai et al. [7] proposed three mathematical models which represent different releasing strategies of sterile mosquitoes. The existence and stability of equilibria were investigated, and the threshold dynamics were obtained. One of the models considered in [7] is

$$
\left\{\begin{array}{l}
\frac{\mathrm{d} w}{\mathrm{~d} t}=\left(\frac{a w}{1+w+g}-\left(\mu_{1}+\xi_{1}(w+g)\right)\right) w, \\
\frac{\mathrm{d} g}{\mathrm{~d} t}=b w-\left(\mu_{2}+\xi_{2}(w+g)\right) g,
\end{array}\right.
$$

where $w$ and $g$ are the numbers of wild mosquitoes and sterile mosquitoes, respectively. All the coefficients are positive constants, $a$ is the number of offspring produced by per mate, $b w$ is the linear release rate of sterile mosquitoes with coefficient $b$, and $\mu_{i}$ and $\xi_{i}(i=1,2)$ represent the density independent and dependent death rates of wild and sterile mosquitoes, respectively. It was shown in $[10,11]$ that continuous model (1) undergoes a sequence of codimensional-1 bifurcations (including saddle-node bifurcation, supercritical and subcritical Hopf bifurcation, and 
homoclinic bifurcation) and codimensional-2 bifurcation (Bogdanov-Takens bifurcation). When $b w$ in (1) is replaced by $b w /(1+w)$, the system becomes a mosquito population model with nonlinear saturated releasing rate of sterile mosquitoes. Some complex bifurcation phenomena, such as Hopf bifurcation of codimension-3, nilpotent cusp of codimension-4, and Bogdanov-Takens bifurcation of codimension-2, were provided in [12] if $\mu_{1} \neq \mu_{2}$ and $\xi_{1} \neq \xi_{2}$.

In mathematical modelling, difference equations (that is, discrete-time models) are also widely used to describe the population dynamics. Compared with continuous models, discrete-time cases have many advantages. The most obvious one is that discrete models are more convenient to formulate and simulate, especially when the populations have nonoverlapping generations [13-15]. Moreover, for several biological models, their discrete-time cases may produce much richer dynamical behaviors than the continuous cases.

In this paper, we study the complex dynamics of the following discrete-time model:

$$
\left\{\begin{array}{l}
w(n+1)=w(n) \exp \left[\frac{a w(n)}{1+w(n)+g(n)}-\left(\mu_{1}+\xi_{1}(w(n)+g(n))\right)\right] \\
g(n+1)=g(n)+b w(n)-\left(\mu_{2}+\xi_{2}(w(n)+g(n))\right) g(n),
\end{array}\right.
$$

which is established from (1) by the Euler forward difference method. $w, g, a, b, \mu_{i}, \xi_{i}(i=1,2)$ are defined as in model (1). By the biological meaning of the model, we only consider system (2) in the region $\Omega=\{(w, g): w \geq 0, g \geq 0\}$ in the $(w, g)$-plane. We will study the existence of codimension-2 bifurcations associated with $1: 2,1: 3$, and $1: 4$ strong resonances of model (2). The codimension-2 bifurcation diagrams, maximum Lyapunov exponent diagrams, and phase portraits are also presented to illustrate the validity of theoretical results and display the complex dynamical behaviors.

The bifurcation and chaotic behavior of discrete models have been widely investigated by many researchers, but most of them focus on codimensional-1 bifurcations, for example, [16-21]. The codimension-2 bifurcations for discrete models are considered very few. The codimension-2 bifurcations of a delayed discrete-time Hopfield neural network, discrete epidemic model with nonlinear incidence rate, and discrete SIS epidemic model with standard incidence are analyzed in [22-24]. A discrete Hindmarsh-Rose model undergoes codimension-2 bifurcations with $1: 2$ and $1: 4$ strong resonances [25], and $1: 3$ strong resonance [26]. 1:1 strong resonance bifurcation of a discrete predator-prey model with nonmonotonic functional response has also been studied in [27]. Moreover, Ren and Yu [28] investigated codimension-2 bifurcations of a discrete information diffusion model.

The main goal of this paper is to investigate the impact of the mating rate $a$ and the release rate coefficient $b$ on the interactive dynamics of wide mosquitoes and sterile mosquitoes. The rest of this paper is organized as follows. Preliminaries are given in Section 2. In Sections 3-5, some sufficient conditions of codimension-2 bifurcations associated with $1: 2,1: 3$, and $1: 4$ strong resonances are established, respectively. The numerical simulations and controller designs are presented in Sections 6 and 7. A brief discussion is given in Section 8.

\section{Preliminaries}

In this section, we first briefly introduce some known results on the existence of positive equilibria, which have been obtained in [7], and then provide some sufficient conditions for the existence of codimension-2 bifurcations with $1: 2$, $1: 3$, and $1: 4$ strong resonances.

Define the function

$$
\begin{aligned}
F(N)= & (1+N)\left(\mu_{1}+\xi_{1} N\right)\left(b+\mu_{2}+\xi_{2} N\right)-a N\left(\mu_{2}+\xi_{2} N\right) \\
= & \xi_{1} \xi_{2} N^{3}+\left(\xi_{1}\left(b+\mu_{2}\right)+\xi_{2}\left(\mu_{1}+\xi_{1}-a\right)\right) N^{2} \\
& +\left(\mu_{1} \xi_{2}+b\left(\mu_{1}+\xi_{1}\right)+\left(\mu_{1}+\xi_{1}-a\right) \mu_{2}\right) N \\
& +\mu_{1}\left(b+\mu_{2}\right) .
\end{aligned}
$$

We can check that model (2) admits a positive equilibrium:

$$
(w, g)=\left(\frac{(1+\bar{N})\left(\mu_{1}+\xi_{1} \bar{N}\right)}{a}, \frac{b(1+\bar{N})\left(\mu_{1}+\xi_{1} \bar{N}\right)}{a\left(\mu_{2}+\xi_{2} \bar{N}\right)}\right),
$$

if and only if $F(N)=0$ has a positive $\operatorname{root} \bar{N}$, which is also equivalent to the existence of positive solution $\bar{N}$ to the equation $G(N)=b$ with

$$
G(N):=\frac{\left(a N-(1+N)\left(\mu_{1}+\xi_{1} N\right)\right)\left(\mu_{2}+\xi_{2} N\right)}{(1+N)\left(\mu_{1}+\xi_{1} N\right)} .
$$

Assuming that $a>\mu_{1}+\xi_{1}+2 \sqrt{\mu_{1} \xi_{1}}$, we know the equation $G(N)=0$ has two positive roots:

$$
N_{1,2}=\frac{1}{2 \xi_{1}}\left(\left(a-\mu_{1}-\xi_{1}\right) \mp \sqrt{\left(a-\mu_{1}-\xi_{1}\right)^{2}-4 \mu_{1} \xi_{1}}\right) .
$$

It follows that there exists a unique $N^{*} \in\left(N_{1}, N_{2}\right)$ such that $G^{\prime}\left(N^{*}\right)=0$. Let

$$
b_{0}:=G\left(N^{*}\right)
$$


then the equation $G(N)=b$ has two positive solutions $N_{1,2}^{*} \in\left(N_{1}, N_{2}\right)$ with $N_{1}^{*}<N_{2}^{*}$ if and only if $b<b_{0}$, and only one positive solution $N^{*} \in\left(N_{1}, N_{2}\right)$ when $b=b_{0}$. In summary, we have the following results on the existence of equilibria of model (2).

Lemma 1. Assume that $a>\mu_{1}+\xi_{1}+2 \sqrt{\mu_{1} \xi_{1}}$. Then,

(i) model (2) always has a boundary equilibrium $E(0,0)$

(ii) if $b>b_{0}$, then model (2) has no any positive equilibrium

(iii) if $b=b_{0}$, then model (2) has a unique positive equilibrium $E^{*}\left(w^{*}, g^{*}\right)$, where

$$
\left(w^{*}, g^{*}\right)=\left(\frac{\left(1+N^{*}\right)\left(\mu_{1}+\xi_{1} N^{*}\right)}{a}, \frac{b_{0}\left(1+N^{*}\right)\left(\mu_{1}+\xi_{1} N^{*}\right)}{a\left(\mu_{2}+\xi_{2} N^{*}\right)}\right) .
$$

$$
\text { Moreover, } F\left(N^{*}\right)=0 \text { and } F^{\prime}\left(N^{*}\right)=0 .
$$

(iv) if $b<b_{0}$, then model (2) has two positive equilibria $E_{1}^{*}\left(w_{1}^{*}, g_{1}^{*}\right)$ and $E_{2}^{*}\left(w_{2}^{*}, g_{2}^{*}\right)$, where

$$
\begin{aligned}
& \left(w_{1}^{*}, g_{1}^{*}\right)=\left(\frac{\left(1+N_{1}^{*}\right)\left(\mu_{1}+\xi_{1} N_{1}^{*}\right)}{a}, \frac{b\left(1+N_{1}^{*}\right)\left(\mu_{1}+\xi_{1} N_{1}^{*}\right)}{a\left(\mu_{2}+\xi_{2} N_{1}^{*}\right)}\right), \\
& \left(w_{2}^{*}, g_{2}^{*}\right)=\left(\frac{\left(1+N_{2}^{*}\right)\left(\mu_{1}+\xi_{1} N_{2}^{*}\right)}{a}, \frac{b\left(1+N_{2}^{*}\right)\left(\mu_{1}+\xi_{1} N_{2}^{*}\right)}{a\left(\mu_{2}+\xi_{2} N_{2}^{*}\right)}\right) .
\end{aligned}
$$

Moreover, $F^{\prime}\left(N_{1}^{*}\right)<0$ and $F^{\prime}\left(N_{2}^{*}\right)>0$.

Denote by $E(w, g)$, the equilibrium of model (2), then the Jacobian matrix at $E(w, g)$ is

$$
J(E)=\left(\begin{array}{cc}
1+w\left(\frac{a(1+g)}{(1+N)^{2}}-\xi_{1}\right) & -w\left(\frac{a w}{(1+N)^{2}}+\xi_{1}\right) \\
b-\xi_{2} g & 1-\mu_{2}-\xi_{2}(N+g)
\end{array}\right),
$$

its trace is $\operatorname{Tr}(J(E))=2+w\left((a 1+g) /(1+N)^{2}-\xi_{1}\right)-\mu_{2}-$ $\xi_{2}(N+g)=2+1 / a A(N)$, and its determinant is $\operatorname{Det}(J(E))=1-w / 1+N F^{\prime}(N)+1 / a A(N)$, where

$$
\begin{aligned}
A(N):= & \xi_{1}\left(\xi_{2}-2 \xi_{1}\right) N^{2}+\left(a\left(\xi_{1}-2 \xi_{2}\right)+\xi_{1}\left(\xi_{2}-\xi_{1}\right)\right. \\
& \left.+\mu_{1}\left(\xi_{2}-3 \xi_{1}\right)\right) N \\
& +a\left(\mu_{1}-\mu_{2}\right)+\mu_{1}\left(\xi_{2}-\xi_{1}-\mu_{1}\right) .
\end{aligned}
$$

If the matrix $J(E)$ has eigenvalues $\lambda_{1,2}=e^{ \pm i \theta}$ with $\theta=\pi$, $\theta=(2 / 3) \pi$, or $\theta=(1 / 2) \pi$, then there may present a codimension-2 bifurcation with $1: 2,1: 3$, or $1: 4$ strong resonances at $E(w, g)$. For the equilibrium $E^{*}\left(w^{*}, g^{*}\right)$ in Lemma 1 (iii), if codimension-2 bifurcation with $1: 2,1: 3$, or $1: 4$ strong resonance occurs, then $\operatorname{Det}\left(J\left(E^{*}\right)\right)=1$ and $\operatorname{Tr}\left(J\left(E^{*}\right)\right)=-2,-1$, or 0 , which imply that $(1 / a) A\left(N^{*}\right)<0$ and $\left(w^{*} /\left(1+N^{*}\right)\right) F^{\prime}\left(N^{*}\right)=(1 / a) A\left(N^{*}\right)<0$. It is a contradiction with $F^{\prime}\left(N^{*}\right)=0$. Similarly, since $F^{\prime}\left(N_{2}^{*}\right)>0$, we can also show that there does not exist the above codimension-2 bifurcations at the equilibrium $E_{2}^{*}\left(w_{2}^{*}, g_{2}^{*}\right)$. Thus, in this paper, we discuss the existence of codimension-2 bifurcation at $E_{1}^{*}\left(w_{1}^{*}, g_{1}^{*}\right)$.

Choosing $a$ and $b$ as bifurcation parameters, if $\left(a, b, \mu_{1}, \mu_{2}, \xi_{1}, \xi_{2}\right) \in F_{1}$, where

$$
\begin{gathered}
F_{1}:=\left\{\left(a, b, \mu_{1}, \mu_{2}, \xi_{1}, \xi_{2}\right) \in \mathbb{R}_{+}^{6}: a>\mu_{1}+\xi_{1}+2 \sqrt{\mu_{1} \xi_{1}},\right. \\
\left.b<b_{0}, \frac{w_{1}^{*}}{1+N_{1}^{*}} F^{\prime}\left(N_{1}^{*}\right)=\frac{1}{a} A\left(N_{1}^{*}\right)=-4\right\},
\end{gathered}
$$

then two eigenvalues of Jacobian matrix $J\left(E_{1}^{*}\right)$ are $\lambda_{1,2}=e^{ \pm \pi i}=-1$. This shows that there may present a codimension-2 bifurcation with $1: 2$ strong resonance at $E_{1}^{*}\left(w_{1}^{*}, g_{1}^{*}\right)$.

If $\left(a, b, \mu_{1}, \mu_{2}, \xi_{1}, \xi_{2}\right) \in F_{2}$, where

$$
\begin{gathered}
F_{2}:=\left\{\left(a, b, \mu_{1}, \mu_{2}, \xi_{1}, \xi_{2}\right) \in \mathbb{R}_{+}^{6}: a>\mu_{1}+\xi_{1}+2 \sqrt{\mu_{1} \xi_{1}},\right. \\
\left.b<b_{0}, \frac{w_{1}^{*}}{1+N_{1}^{*}} F^{\prime}\left(N_{1}^{*}\right)=\frac{1}{a} A\left(N_{1}^{*}\right)=-3\right\},
\end{gathered}
$$

then two eigenvalues of the Jacobian matrix $J\left(E_{1}^{*}\right)$ are $\lambda_{1,2}=e^{ \pm(2 \pi / 3) i}=-(1 / 2) \pm(\sqrt{3} / 2) i$. This shows that there may present a codimension-2 bifurcation with $1: 3$ strong resonance at $E_{1}^{*}\left(w_{1}^{*}, g_{1}^{*}\right)$.

Furthermore, if $\left(a, b, \mu_{1}, \mu_{2}, \xi_{1}, \xi_{2}\right) \in F_{3}$, where

$$
\begin{gathered}
F_{3}:=\left\{\left(a, b, \mu_{1}, \mu_{2}, \xi_{1}, \xi_{2}\right) \in \mathbb{R}_{+}^{6}: a>\mu_{1}+\xi_{1}+2 \sqrt{\mu_{1} \xi_{1}},\right. \\
\left.b<b_{0}, \frac{w_{1}^{*}}{1+N_{1}^{*}} F^{\prime}\left(N_{1}^{*}\right)=\frac{1}{a} A\left(N_{1}^{*}\right)=-2\right\},
\end{gathered}
$$

then two eigenvalues of the Jacobian matrix $J\left(E_{1}^{*}\right)$ are $\lambda_{1,2}=e^{ \pm(\pi / 2) i}= \pm i$. This shows that there may present a codimension-2 bifurcation with $1: 4$ strong resonance at $E_{1}^{*}\left(w_{1}^{*}, g_{1}^{*}\right)$. In the following, we will give the theoretical analysis and numerical simulations.

\section{1 : 2 Strong Resonance}

In this section, by applying the bifurcation theory in [29], we analyze the codimension-2 bifurcation associated with $1: 2$ strong resonance of model (2) at the equilibrium $E_{1}^{*}\left(w_{1}^{*}, g_{1}^{*}\right)$.

We choose $a$ and $b$ as bifurcation parameters and assume that $\left(a_{1}, b_{1}, \mu_{1}, \mu_{2}, \xi_{1}, \xi_{2}\right) \in F_{1}$. Consider model (2) with $a=$ $a_{1}+a_{1}^{*}$ and $b=b_{1}+b_{1}^{*}$, where $a_{1}^{*}$ and $b_{1}^{*}$ are small perturbations of the parameters $a_{1}$ and $b_{1}$. Let $\widehat{w}(n)=w(n)-$ $w_{1}^{*}$ and $\hat{g}(n)=g(n)-g_{1}^{*}$, and model (2) becomes into the following form which has the equilibrium $(0,0)$ : 


$$
\left\{\begin{array}{l}
\widehat{w}(n+1)=\left(\widehat{w}(n)+w_{1}^{*}\right) \exp \left[\frac{a\left(\widehat{w}(n)+w_{1}^{*}\right)}{1+\widehat{w}(n)+\widehat{g}(n)+w_{1}^{*}+g_{1}^{*}}-\left(\mu_{1}+\xi_{1}\left(\widehat{w}(n)+\widehat{g}(n)+w_{1}^{*}+g_{1}^{*}\right)\right)\right]-w_{1}^{*} \\
\widehat{g}(n+1)=\widehat{g}(n)+b\left(\widehat{w}(n)+w_{1}^{*}\right)-\left(\mu_{2}+\xi_{2}\left(\widehat{w}(n)+\widehat{g}(n)+w_{1}^{*}+g_{1}^{*}\right)\right)\left(\widehat{g}(n)+g_{1}^{*}\right)
\end{array}\right.
$$

By expanding the right-hand side of (15) into the Taylor series at $(0,0)$, we have

$$
\left\{\begin{array}{l}
\widehat{w}(n+1)=\left(1+w_{1}^{*} \alpha\right) \widehat{w}(n)-w_{1}^{*} \beta \widehat{g}(n)+\sum_{2 \leq i+j \leq 3} k_{i j}(a, b) \widehat{w}^{i}(n) \widehat{g}^{j}(n)+O\left((|\widehat{w}(n)|+|\widehat{g}(n)|)^{4}\right) \\
\widehat{g}(n+1)=\left(b-\xi_{2} g_{1}^{*}\right) \widehat{w}(n)+\left[1-\left(\mu_{2}+\xi_{2} g_{1}^{*}+\xi_{2} N_{1}^{*}\right)\right] \widehat{g}(n)-\xi_{2} \widehat{w}(n) \widehat{g}(n)-\xi_{2} \widehat{g}^{2}(n)+O\left((|\widehat{w}(n)|+|\widehat{g}(n)|)^{4}\right)
\end{array}\right.
$$

where

$$
\begin{aligned}
& \alpha=\frac{a\left(1+g_{1}^{*}\right)}{\left(1+N_{1}^{*}\right)^{2}}-\xi_{1} \\
& \beta=\frac{a w_{1}^{*}}{\left(1+N_{1}^{*}\right)^{2}}+\xi_{1} \text {, } \\
& k_{20}(a, b)=\alpha+\frac{1}{2} w_{1}^{*} \alpha^{2}-\frac{w_{1}^{*}}{1+N_{1}^{*}}\left(\alpha+\xi_{1}\right) \text {, } \\
& k_{11}(a, b)=-\beta-w_{1}^{*} \alpha \beta-\frac{a w_{1}^{*}}{\left(1+N_{1}^{*}\right)^{2}}+\frac{2 a\left(w_{1}^{*}\right)^{2}}{\left(1+N_{1}^{*}\right)^{3}}, \\
& k_{02}(a, b)=\frac{1}{2} w_{1}^{*} \beta^{2}+\frac{a\left(w_{1}^{*}\right)^{2}}{\left(1+N_{1}^{*}\right)^{3}} \text {, } \\
& k_{30}(a, b)=\frac{1}{2} \alpha^{2}+\frac{1}{6} w_{1}^{*} \alpha^{3}-\frac{w_{1}^{*}}{1+N_{1}^{*}} \alpha\left(\alpha+\xi_{1}\right)-\frac{1}{a}\left(\alpha+\xi_{1}\right)^{2} \text {, } \\
& k_{03}(a, b)=-\frac{1}{6} w_{1}^{*} \beta^{3}-\frac{a\left(w_{1}^{*}\right)^{2}}{\left(1+N_{1}^{*}\right)^{3}} \beta-\frac{a\left(w_{1}^{*}\right)^{2}}{\left(1+N_{1}^{*}\right)^{4}}, \\
& k_{21}(a, b)=-\alpha \beta-\frac{1}{2} w_{1}^{*} \alpha^{2} \beta+\frac{a w_{1}^{*}\left(1+g_{1}^{*}\right)}{\left(1+N_{1}^{*}\right)^{3}} \beta \\
& -\frac{a w_{1}^{*}\left(2+g_{1}^{*}-3 w_{1}^{*}\right)}{2\left(1+N_{1}^{*}\right)^{3}} \alpha \\
& -\frac{a}{\left(1+N_{1}^{*}\right)^{2}}+\frac{4 a w_{1}^{*}}{\left(1+N_{1}^{*}\right)^{3}}-\frac{3 a\left(w_{1}^{*}\right)^{2}}{\left(1+N_{1}^{*}\right)^{4}} \\
& k_{12}(a, b)=\frac{a w_{1}^{*}\left(1+g_{1}^{*}-w_{1}^{*}\right)}{\left(1+N_{1}^{*}\right)^{3}} \beta+\frac{a\left(w_{1}^{*}\right)^{2}}{\left(1+N_{1}^{*}\right)^{3}} \alpha+\frac{1}{2} \beta^{2} \\
& +\frac{1}{2} w_{1}^{*} \alpha \beta^{2} \\
& +\frac{a w_{1}^{*}\left(2+2 N_{1}^{*}-3 w_{1}^{*}\right)}{\left(1+N_{1}^{*}\right)^{4}}
\end{aligned}
$$
is

For $\left(a_{1}, b_{1}, \mu_{1}, \mu_{2}, \xi_{1}, \xi_{2}\right) \in F_{1}$, the Jacobian matrix at $E_{1}^{*}$

$$
J\left(E_{1}^{*}\right)=\left(\begin{array}{cc}
1+w_{1}^{*} \alpha_{1} & -w_{1}^{*} \beta_{1} \\
b_{1}-\xi_{2} g_{1}^{*} & 1-\mu_{2}-\xi_{2}\left(N_{1}^{*}+g_{1}^{*}\right)
\end{array}\right),
$$

where

$$
\begin{aligned}
& \alpha_{1}:=\frac{a_{1}\left(1+g_{1}^{*}\right)}{\left(1+N_{1}^{*}\right)^{2}}-\xi_{1}, \\
& \beta_{1}:=\frac{a_{1} w_{1}^{*}}{\left(1+N_{1}^{*}\right)^{2}}+\xi_{1} .
\end{aligned}
$$

Take two linearly independent (generalized) eigenvectors of $J\left(E_{1}^{*}\right)$,

$$
\begin{aligned}
& p_{1}=\left(\begin{array}{c}
\frac{\beta_{1} w_{1}^{*}}{2+\alpha_{1} w_{1}^{*}} \\
1
\end{array}\right), \\
& p_{2}=\left(\begin{array}{c}
\frac{1}{b_{1}-\xi_{2} g_{1}^{*}} \\
0
\end{array}\right),
\end{aligned}
$$

and that of the transposed matrix $J^{T}\left(E_{1}^{*}\right)$,

$$
\begin{aligned}
& q_{1}=\left(\begin{array}{c}
b_{1}-\xi_{2} g_{1}^{*} \\
-\left(2+\alpha_{1} w_{1}^{*}\right)
\end{array}\right), \\
& q_{2}=\left(\begin{array}{l}
0 \\
1
\end{array}\right) .
\end{aligned}
$$

It is easy to check that $\left\langle p_{1}, q_{2}\right\rangle=\left\langle p_{2}, q_{1}\right\rangle=1,\left\langle p_{1}, q_{1}\right\rangle=\left\langle p_{2}, q_{2}\right\rangle=0$, where $\langle x, y\rangle:=x^{T} y=x_{1} y_{1}+x_{2} y_{2}$ for any $x=\left(x_{1}, x_{2}\right)^{T}$, $y=\left(y_{1}, y_{2}\right)^{T} \in \mathbb{R}^{2}$. Therefore, any vector $(\widehat{w}(n)$, $\widehat{g}(n))^{T} \in \mathbb{R}^{2}$ can be represented in the form

$$
(\widehat{w}(n), \widehat{g}(n))^{T}=x(n) p_{1}+y(n) p_{2},
$$


which implies that

$$
\left\{\begin{array}{l}
x(n)=\left\langle(\widehat{w}(n), \widehat{g}(n))^{T}, q_{2}\right\rangle, \\
y(n)=\left\langle(\widehat{w}(n), \widehat{g}(n))^{T}, q_{1}\right\rangle .
\end{array}\right.
$$

Under new coordinates $(x(n), y(n))$, map (16) can be written as

$$
\left\{\begin{array}{l}
x(n+1)=\left(-1+\Theta_{1}(a, b)\right) x(n)+\left(1-\frac{1}{2} \Theta_{1}(a, b)\right) y(n)-\xi_{2}\left(1+\frac{\beta_{1} w_{1}^{*}}{2+\alpha_{1} w_{1}^{*}}\right) x^{2}(n) \\
-\frac{\xi_{2}}{b_{1}-\xi_{2} g_{1}^{*}} x(n) y(n)+O\left((|x(n)|+|y(n)|)^{4}\right), \\
y(n+1)=\Theta_{2}(a, b) x(n)+\left(-1-\frac{1}{2} \Theta_{2}(a, b)\right) y(n)+\sum_{2 \leq i+j \leq 3} r_{i j}(a, b) x^{i}(n) y^{j}(n) \\
+O\left((|x(n)|+|y(n)|)^{4}\right)
\end{array}\right.
$$

where

$$
\begin{aligned}
& \Theta_{1}(a, b)=2\left(1-\frac{b-\xi_{2} g_{1}^{*}}{b_{1}-\xi_{2} g_{1}^{*}}\right) \\
& \Theta_{2}(a, b)=-2\left[\left(2+w_{1}^{*} \alpha\right)-\left(2+w_{1}^{*} \alpha_{1}\right) \frac{b-\xi_{2} g_{1}^{*}}{b_{1}-\xi_{2} g_{1}^{*}}\right], \\
& r_{20}=\left(b_{1}-\xi_{2} g_{1}^{*}\right)\left[k_{11} \frac{\beta_{1} w_{1}^{*}}{2+\alpha_{1} w_{1}^{*}}+k_{20}\left(\frac{\beta_{1} w_{1}^{*}}{2+\alpha_{1} w_{1}^{*}}\right)^{2}+k_{02}\right] \\
& +\left(2+\alpha_{1} w_{1}^{*}\right) \xi_{2}+\beta_{1} w_{1}^{*} \xi_{2}, \\
& r_{02}=\frac{k_{20}}{b_{1}-\xi_{2} g_{1}^{*}}, \\
& r_{11}=k_{11}+2 k_{20} \frac{\beta_{1} w_{1}^{*}}{2+\alpha_{1} w_{1}^{*}}+\frac{\left(2+\alpha_{1} w_{1}^{*}\right) \xi_{2}}{b_{1}-\xi_{2} g_{1}^{*}}, \\
& r_{30}=\left(b_{1}-\xi_{2} g_{1}^{*}\right)\left[k_{30}\left(\frac{\beta_{1} w_{1}^{*}}{2+\alpha_{1} w_{1}^{*}}\right)^{3}+k_{21}\left(\frac{\beta_{1} w_{1}^{*}}{2+\alpha_{1} w_{1}^{*}}\right)^{2}\right. \\
& \left.+k_{12} \frac{\beta_{1} w_{1}^{*}}{2+\alpha_{1} w_{1}^{*}}+k_{03}\right] \\
& r_{21}=3 k_{30}\left(\frac{\beta_{1} w_{1}^{*}}{2+\alpha_{1} w_{1}^{*}}\right)^{2}+2 k_{21} \frac{\beta_{1} w_{1}^{*}}{2+\alpha_{1} w_{1}^{*}}+k_{12} \\
& r_{12}=3 k_{30} \frac{\beta_{1} w_{1}^{*}}{\left(2+\alpha_{1} w_{1}^{*}\right)\left(b_{1}-\xi_{2} g_{1}^{*}\right)}+k_{21} \frac{1}{b_{1}-\xi_{2} g_{1}^{*}}, \\
& r_{03}=k_{30}\left(\frac{1}{b_{1}-\xi_{2} g_{1}^{*}}\right)^{2} .
\end{aligned}
$$

By introducing a coordinate transformation

$$
\left(\begin{array}{l}
x(n) \\
y(n)
\end{array}\right)=\left(\begin{array}{cc}
1-\frac{1}{2} \Theta_{1}(a, b) & 0 \\
-\Theta_{1}(a, b) & 1
\end{array}\right)\left(\begin{array}{c}
\hat{x}(n) \\
\hat{y}(n)
\end{array}\right),
$$

map (24) can be reduced into the following form:

$$
\left\{\begin{array}{l}
\hat{x}(n+1)=-\widehat{x}(n)+\widehat{y}(n)+\widehat{k}_{20} \widehat{x}^{2}(n)+\widehat{k}_{11} \widehat{x}(n) \widehat{y}(n) \\
+O\left((|\widehat{x}(n)|+|\widehat{y}(n)|)^{4}\right), \\
\hat{y}(n+1)=\Theta_{2} \hat{x}(n)+\left(-1+\Theta_{3}\right) \hat{y}(n)+\sum_{2 \leq i+j \leq 3} \widehat{r}_{i j}(a, b) \hat{x}^{i}(n) \widehat{y}^{j}(n) \\
+O\left((|\widehat{x}(n)|+|\widehat{y}(n)|)^{4}\right),
\end{array}\right.
$$

where

$$
\begin{aligned}
& \Theta_{3}(a, b)=\Theta_{1}(a, b)-\frac{1}{2} \Theta_{2}(a, b), \\
& \widehat{k}_{11}(k, r)=-\frac{\xi_{2}}{b_{1}-\xi_{2} g_{1}^{*}}, \\
& \widehat{r}_{20}(a, b)=\Theta_{1} \widehat{k}_{20}+\left(1-\frac{\Theta_{1}}{2}\right)^{2} r_{20}+\Theta_{1}^{2} r_{02}-\left(1-\frac{\Theta_{1}}{2}\right) \Theta_{1} r_{11}, \\
& \widehat{k}_{20}(k, r)=-\left(1-\frac{\Theta_{1}}{2}\right) \xi_{2}\left(1+\frac{\beta_{1} w_{1}^{*}}{2+\alpha_{1} w_{1}^{*}}\right)+\Theta_{1} \frac{\xi_{2}}{b_{1}-\xi_{2} g_{1}^{*}}, \\
& \widehat{r}_{02}(a, b)=r_{02},
\end{aligned}
$$$$
\widehat{r}_{11}(a, b)=\Theta_{1} \widehat{k}_{11}+\left(1-\frac{\Theta_{1}}{2}\right) r_{11}-2 \Theta_{1} r_{02}
$$ 


$$
\begin{aligned}
\widehat{r}_{30}(a, b)= & \left(1-\frac{\Theta_{1}}{2}\right)^{3} r_{30}-\Theta_{1}^{3} r_{03}-\Theta_{1}\left(1-\frac{\Theta_{1}}{2}\right)^{2} r_{21} \\
& +\Theta_{1}^{2}\left(1-\frac{\Theta_{1}}{2}\right) r_{12}, \\
\widehat{r}_{21}(a, b)= & 3 \Theta_{1}^{2} r_{03}+\left(1-\frac{\Theta_{1}}{2}\right)^{2} r_{21}-2 \Theta_{1}\left(1-\frac{\Theta_{1}}{2}\right) r_{12}, \\
\widehat{r}_{12}(a, b)= & \left(1-\frac{\Theta_{1}}{2}\right) r_{12}-3 \Theta_{1} r_{03}, \\
\widehat{r}_{03}(a, b)= & r_{03} .
\end{aligned}
$$

Next, we aim to eliminate all quadratic terms and some cubic terms in (27). To achieve it, we introduce the following transformation:

$$
\left\{\begin{array}{l}
\hat{x}(n)=u(n)+\sum_{2 \leq i+j \leq 3} \varphi_{i j}(a, b) u^{i}(n) v^{j}(n), \\
\widehat{y}(n)=v(n)+\sum_{2 \leq i+j \leq 3} \psi_{i j}(a, b) u^{i}(n) v^{j}(n),
\end{array}\right.
$$

where $\varphi_{i j}$ and $\psi_{i j}(2 \leq i+j \leq 3)$ will be determined later.

By applying transformation (29) and its inverse, map (27) becomes

$$
\left\{\begin{array}{l}
u(n+1)=-u(n)+v(n)+\sum_{2 \leq i+j \leq 3} \eta_{i j}(a, b) u^{i}(n) v^{j}(n) \\
\quad+O\left((|u(n)|+|v(n)|)^{4}\right) \\
v(n+1)=\Theta_{2}(a, b) u(n)+\left(-1+\Theta_{3}(a, b)\right) v(n) \\
\quad+\sum_{2 \leq i+j \leq 3} \zeta_{i j}(a, b) u^{i}(n) v^{j}(n)+O\left((|u(n)|+|v(n)|)^{4}\right)
\end{array}\right.
$$

where

$$
\begin{aligned}
\eta_{20}(a, b)= & \widehat{k}_{20}+\psi_{20}-2 \varphi_{20}-\Theta_{2}^{2} \varphi_{02}+\Theta_{2} \varphi_{11}, \\
\eta_{11}(a, b)= & \widehat{k}_{11}+\psi_{11}-2 \Theta_{2}\left(1+\Theta_{3}\right) \varphi_{02} \\
& +\left(\Theta_{3}-\Theta_{2}\right) \varphi_{11}+2 \varphi_{20} \\
\eta_{02}(a, b)= & \psi_{02}-\left(1+\left(1+\Theta_{3}\right)^{2}\right) \varphi_{02}+\left(1+\Theta_{3}\right) \varphi_{11}-\varphi_{20}, \\
\zeta_{20}(a, b)= & \widehat{r}_{20}-\Theta_{2}^{2} \psi_{02}+\Theta_{2} \psi_{11}+\left(\Theta_{2}+\Theta_{3}\right) \psi_{20}, \\
\zeta_{11}(a, b)= & \widehat{r}_{11}-2 \Theta_{2}\left(1+\Theta_{3}\right) \psi_{02}+\left(2-\Theta_{2}+2 \Theta_{3}\right) \psi_{11} \\
& +2 \psi_{20}+\Theta_{2} \varphi_{11}, \\
\zeta_{02}(a, b)= & \widehat{r}_{20}-\Theta_{3}\left(1+\Theta_{3}\right) \psi_{02}-\left(1+\Theta_{3}\right) \psi_{11}-\psi_{20}+\Theta_{2} \varphi_{02} .
\end{aligned}
$$

Assume that the following vanishing conditions hold:

$$
\eta_{20}=\eta_{11}=\eta_{02}=\zeta_{20}=\zeta_{11}=\zeta_{02}=0
$$

and we can obtain all the coefficients $\varphi_{i j}$ and $\psi_{i j}$ satisfying $i+j=2$. Moreover, by taking $\eta_{30}=\eta_{12}=\eta_{21}=\eta_{03}$ $=\zeta_{12}=\zeta_{03}=0$, we can determine the coefficients $\varphi_{i j}$ and $\psi_{i j}$ for $i+j=3$, see more details in [24, 25]. Thus, model (15) can be transformed into the normal form for $1: 2$ strong resonance bifurcation as follows:

$$
\left\{\begin{array}{l}
u(n+1)=-u(n)+v(n)+O\left((|u(n)|+|v(n)|)^{4}\right) \\
v(n+1)=\Theta_{2}(a, b) u(n)+\left(-1+\Theta_{3}(a, b)\right) v(n)+C(a, b) u^{3}(n) \\
+D(a, b) u^{2}(n) v(n)+O\left((|u(n)|+|v(n)|)^{4}\right)
\end{array}\right.
$$

where $C(a, b)$ and $D(a, b)$ satisfy

$$
\begin{aligned}
C\left(a_{1}, b_{1}\right)= & \widehat{r}_{30}\left(a_{1}, b_{1}\right)+\widehat{k}_{20}\left(a_{1}, b_{1}\right) \widehat{r}_{20}\left(a_{1}, b_{1}\right)+\frac{1}{2} \widehat{r}_{20}^{2}\left(a_{1}, b_{1}\right)+\frac{1}{2} \widehat{r}_{20}\left(a_{1}, b_{1}\right) \widehat{r}_{11}\left(a_{1}, b_{1}\right), \\
D\left(a_{1}, b_{1}\right)= & \widehat{r}_{21}\left(a_{1}, b_{1}\right)+\frac{1}{2} \widehat{k}_{20}\left(a_{1}, b_{1}\right) \widehat{r}_{11}\left(a_{1}, b_{1}\right)+\frac{5}{4} \widehat{r}_{20}\left(a_{1}, b_{1}\right) \widehat{r}_{11}\left(a_{1}, b_{1}\right) \\
& +\widehat{r}_{20}\left(a_{1}, b_{1}\right) \widehat{r}_{02}\left(a_{1}, b_{1}\right)+3 \widehat{k}_{20}^{2}\left(a_{1}, b_{1}\right)+\frac{5}{2} \widehat{k}_{20}\left(a_{1}, b_{1}\right) \widehat{r}_{20}\left(a_{1}, b_{1}\right) \\
& +\frac{5}{2} \widehat{k}_{11}\left(a_{1}, b_{1}\right) \widehat{r}_{20}\left(a_{1}, b_{1}\right)+\widehat{r}_{20}^{2}\left(a_{1}, b_{1}\right)+\frac{1}{2} \widehat{r}_{11}^{2}\left(a_{1}, b_{1}\right) .
\end{aligned}
$$


Applying the results established in [29], we can get the existence of $1: 2$ strong resonance bifurcation.

Theorem 1. Assume that $C\left(a_{1}, b_{1}\right) \neq 0$ and $D\left(a_{1}, b_{1}\right)+$ $3 C\left(a_{1}, b_{1}\right) \neq 0$. Then, model (2) has a $1: 2$ strong resonance bifurcation at $E_{1}^{*}\left(w_{1}^{*}, g_{1}^{*}\right)$ when the parameters $a$ and $b$ vary in a sufficiently small neighborhood of $F_{1}$ with fixed $\mu_{1}, \mu_{2}, \xi_{1}$, and $\xi_{2}$. If we further assume $C\left(a_{1}, b_{1}\right)<0$, then $E_{1}^{*}\left(w_{1}^{*}, g_{1}^{*}\right)$ is a saddle; if $C\left(a_{1}, b_{1}\right)>0$, then $E_{1}^{*}\left(w_{1}^{*}, g_{1}^{*}\right)$ is elliptic. $D\left(a_{1}, b_{1}\right)+3 C\left(a_{1}, b_{1}\right)$ determines the bifurcation scenario under small perturbations. Moreover, model (2) has the following bifurcation behaviors:

(i) There is a pitchfork bifurcation curve $P=\left\{\left(\theta_{1}, \theta_{2}\right): \theta_{1}=0\right\}$, and there exist nontrivial equilibria for $\theta_{1}<0$

(ii) There is a nondegenerate Neimark-Sacker bifurcation curve $H=\left\{\left(\theta_{1}, \theta_{2}\right): \theta_{1}=-\theta_{2}+O\left(\left(\left|\theta_{1}\right|+\left|\theta_{2}\right|\right)^{2}\right), \theta_{1}<0\right\}$

(iii) There is a heteroclinic bifurcation curve $H L=$$$
\left\{\left(\theta_{1}, \theta_{2}\right): \theta_{1}=-(5 / 3) \theta_{2}+O\left(\left(\left|\theta_{1}\right|+\left|\theta_{2}\right|\right)^{2}\right), \theta_{1}<0\right\}
$$

\section{1 : 3 Strong Resonance}

In this section, we prove that there exists a codimension-2 bifurcation with $1: 3$ strong resonance of model (2) at $E_{1}^{*}\left(w_{1}^{*}, g_{1}^{*}\right)$. We choose $a$ and $b$ as bifurcation parameters of model (2) and assume that $\left(a_{2}, b_{2}, \mu_{1}, \mu_{2}, \xi_{1}, \xi_{2}\right) \in F_{2}$.

Similar to Section 3, we can also transform $E_{1}^{*}\left(w_{1}^{*}, g_{1}^{*}\right)$ into $(0,0)$ and then expand the right-hand side of the map into Taylor series. For $\left(a_{2}, b_{2}, \mu_{1}, \mu_{2}, \xi_{1}, \xi_{2}\right) \in F_{2}$, the Jacobian matrix at $E_{1}^{*}\left(w_{1}^{*}, g_{1}^{*}\right)$ is

$$
J\left(E_{1}^{*}\right)=\left(\begin{array}{cc}
1+w_{1}^{*} \alpha_{2} & -w_{1}^{*} \beta_{2} \\
b_{2}-\xi_{2} g_{1}^{*} & 1-\mu_{2}-\xi_{2}\left(N_{1}^{*}+g_{1}^{*}\right)
\end{array}\right),
$$

with

$$
\begin{aligned}
& \alpha_{2}:=\frac{a_{2}\left(1+g_{1}^{*}\right)}{\left(1+N_{1}^{*}\right)^{2}}-\xi_{1}, \\
& \beta_{2}:=\frac{a_{2} w_{1}^{*}}{\left(1+N_{1}^{*}\right)^{2}}+\xi_{1},
\end{aligned}
$$

and it has two complex eigenvalues $\lambda_{1,2}=-(1 / 2) \pm(\sqrt{3} / 2) i$. The corresponding eigenvector $\zeta_{1} \in \mathbb{C}^{2}$ and adjoint eigenvector $\zeta_{2} \in \mathbb{C}^{2}$ of $J\left(E_{1}^{*}\right)$ are defined as

$$
\begin{aligned}
& \zeta_{1}=\left(\begin{array}{c}
x_{1} \\
y_{1}
\end{array}\right):=\left(\begin{array}{c}
w_{1}^{*} \beta_{2} \\
\frac{3}{2}+w_{1}^{*} \alpha_{2}-\frac{\sqrt{3}}{2} i
\end{array}\right), \\
& \zeta_{2}=\left(\begin{array}{c}
x_{2} \\
y_{2}
\end{array}\right):=\left(\begin{array}{c}
\frac{3+\sqrt{3}\left(3+2 w_{1}^{*} \alpha_{2}\right) i}{6 w_{1}^{*} \beta_{2}} \\
-\frac{\sqrt{3}}{3} i
\end{array}\right) .
\end{aligned}
$$

Any vector $Z(n)=(\widehat{w}(n), \widehat{g}(n))^{T} \in \mathbb{R}^{2}$ can be represented as $Z(n)=z(n) \zeta_{1}+\bar{z}(n) \bar{\zeta}_{1}$. Thus, for $\left(a_{2}, b_{2}, \mu_{1}, \mu_{2}, \xi_{1}, \xi_{2}\right) \in F_{2}$, model (16) can be written in the complex form as follows:

$$
z(n+1)=\lambda_{1} z(n)+\sum_{2 \leq i+j \leq 3} \frac{\sigma_{i j}}{i ! j !} z^{i}(n) \bar{z}^{j}(n)+O\left(|z(n)|^{4}\right),
$$

where $\lambda_{1}=-(1 / 2)+(\sqrt{3} / 2) i$ and

$$
\begin{aligned}
\sigma_{20}= & 2 \bar{x}_{2}\left(k_{20}\left(a_{2}, b_{2}\right) x_{1}^{2}+k_{11}\left(a_{2}, b_{2}\right) x_{1} y_{1}+k_{02}\left(a_{2}, b_{2}\right) y_{1}^{2}\right) \\
& -2 \xi_{2} \bar{y}_{2}\left(x_{1} y_{1}+y_{1}^{2}\right), \\
\sigma_{02}= & 2 \bar{x}_{2}\left(k_{20}\left(a_{2}, b_{2}\right) \bar{x}_{1}^{2}+k_{11}\left(a_{2}, b_{2}\right) \bar{x}_{1} \bar{y}_{1}+k_{02}\left(a_{2}, b_{2}\right) \bar{y}_{1}^{2}\right) \\
& -2 \xi_{2} \bar{y}_{2}\left(\bar{x}_{1} \bar{y}_{1}+\bar{y}_{1}^{2}\right),
\end{aligned}
$$$$
\sigma_{11}=\bar{x}_{2}\left[2 k_{20}\left(a_{2}, b_{2}\right) x_{1} \bar{x}_{1}+k_{11}\left(a_{2}, b_{2}\right)\left(x_{1} \bar{y}_{1}+\bar{x}_{1} y_{1}\right)\right.
$$$$
\left.+2 k_{02}\left(a_{2}, b_{2}\right) y_{1} \bar{y}_{1}\right]
$$$$
-\xi_{2} \bar{y}_{2}\left(x_{1} \bar{y}_{1}+\bar{x}_{1} y_{1}+2 y_{1} \bar{y}_{1}\right)
$$$$
\sigma_{30}=6 \bar{x}_{2}\left(k_{30}\left(a_{2}, b_{2}\right) x_{1}^{3}+k_{21}\left(a_{2}, b_{2}\right) x_{1}^{2} y_{1}+k_{12}\left(a_{2}, b_{2}\right) x_{1} y_{1}^{2}\right.
$$$$
\left.+k_{03}\left(a_{2}, b_{2}\right) y_{1}^{3}\right) \text {, }
$$$$
\sigma_{03}=6 \bar{x}_{2}\left(k_{30}\left(a_{2}, b_{2}\right) \bar{x}_{1}^{3}+k_{21}\left(a_{2}, b_{2}\right) \bar{x}_{1}^{2} \bar{y}_{1}+k_{12}\left(a_{2}, b_{2}\right) \bar{x}_{1} \bar{y}_{1}^{2}\right.
$$$$
\left.+k_{03}\left(a_{2}, b_{2}\right) \bar{y}_{1}^{3}\right) \text {, }
$$$$
\sigma_{21}=2 \bar{x}_{2}\left[3 k_{30}\left(a_{2}, b_{2}\right) x_{1}^{2} \bar{x}_{1}+k_{21}\left(a_{2}, b_{2}\right) x_{1}^{2} \bar{y}_{1}\right.
$$

$$
\begin{aligned}
& +2 k_{21}\left(a_{2}, b_{2}\right) x_{1} \bar{x}_{1} y_{1} \\
& \left.+k_{12}\left(a_{2}, b_{2}\right)\left(y_{1}^{2} \bar{x}_{1}+2 x_{1} y_{1} \bar{y}_{1}\right)+3 k_{03}\left(a_{2}, b_{2}\right) y_{1}^{2} \bar{y}_{1}\right], \\
\sigma_{12}= & 2 \bar{x}_{2}\left[3 k_{30}\left(a_{2}, b_{2}\right) x_{1} \bar{x}_{1}^{2}+k_{21}\left(a_{2}, b_{2}\right) \bar{x}_{1}^{2} y_{1}\right. \\
& +2 k_{21}\left(a_{2}, b_{2}\right) x_{1} \bar{x}_{1} \bar{y}_{1}
\end{aligned}
$$

Next, we shall establish the normal form for $1: 3$ strong resonance bifurcation. We first eliminate some quadratic terms in (38) through the transformation

$$
z(n)=\phi(n)+\frac{h_{20}}{2} \phi^{2}(n)+h_{11} \phi(n) \bar{\phi}(n)+\frac{h_{02}}{2} \bar{\phi}^{2}(n),
$$

where the coefficients $h_{i j}$ satisfying $i+j=2$ will be determined later.

Using transformation (40) and its inverse transformation, 


$$
\begin{aligned}
\phi(n)= & z(n)-\frac{h_{20}}{2} z^{2}(n)-h_{11} z(n) \bar{z}(n)-\frac{h_{02}}{2} \bar{z}^{2}(n) \\
& +\frac{1}{2}\left(h_{20}^{2}+h_{11} \bar{h}_{02}\right) z^{3}(n) \\
& +\left(\left|h_{11}\right|^{2}+\frac{3}{2} h_{11} h_{20}+\frac{1}{2}\left|h_{02}\right|^{2}\right) z^{2}(n) \bar{z}(n) \\
& +\left(h_{02} \bar{h}_{11}+h_{11}^{2}+\frac{1}{2} h_{11} \bar{h}_{20}+\frac{1}{2} h_{20} h_{02}\right) z(n) \bar{z}^{2}(n) \\
& +\frac{1}{2}\left(h_{11} h_{02}+h_{02} \bar{h}_{20}\right) \bar{z}^{3}(n)+O\left(|z(n)|^{4}\right),
\end{aligned}
$$

map (41) is changed into the following form:

$$
\phi(n+1)=\lambda_{1} \phi(n)+\sum_{2 \leq i+j \leq 3} \frac{\widehat{\sigma}_{i j}}{i ! j !} \phi^{i}(n) \bar{\phi}^{j}(n)+O\left(|\phi(n)|^{4}\right),
$$

where

$$
\begin{aligned}
& \widehat{\sigma}_{20}=\sqrt{3} i h_{20}+\sigma_{20} \text {, } \\
& \widehat{\sigma}_{11}=\left(-\frac{3}{2}+\frac{\sqrt{3}}{2} i\right) h_{11}+\sigma_{11} \text {, } \\
& \widehat{\sigma}_{02}=\sigma_{02} \text {, } \\
& \widehat{\sigma}_{30}=\frac{9-3 \sqrt{3} i}{2} \sigma_{20} h_{20}+3 \sigma_{11} \bar{h}_{02}+\sigma_{30}+\frac{9+3 \sqrt{3} i}{2} h_{20}^{2} \\
& -3 \lambda_{1} \bar{\sigma}_{02} h_{11} \text {, } \\
& \widehat{\sigma}_{21}=2 \sigma_{11} \bar{h}_{11}+\sigma_{11} h_{20}+\sigma_{02} \bar{h}_{02}+\sigma_{21}+(1-\sqrt{3} i) \sigma_{11} h_{20} \\
& +\frac{1+\sqrt{3} i}{2} \bar{\sigma}_{02} h_{02} \\
& +(-3+\sqrt{3} i)\left|h_{11}\right|^{2}+\frac{-3+5 \sqrt{3} i}{2} h_{11} h_{20} \\
& +(1-\sqrt{3} i) h_{11} \bar{\sigma}_{11}+\frac{5+\sqrt{3} i}{2} \sigma_{20} h_{11} \text {, } \\
& \widehat{\sigma}_{12}=(3+\sqrt{3} i) \sigma_{11} h_{11}+\sigma_{11} \bar{h}_{20}+2 \sigma_{02} \bar{h}_{11}+\sigma_{20} h_{02}+\sigma_{12} \\
& +(1-\sqrt{3} i) \sigma_{02} h_{20} \\
& -(3+\sqrt{3} i) h_{11}^{2}-\frac{3+\sqrt{3} i}{2} h_{11} \bar{h}_{20}+\frac{1-\sqrt{3} i}{2} h_{11} \bar{\sigma}_{20} \\
& -2 \sqrt{3} i h_{02} \bar{h}_{11}+(1+\sqrt{3} i) \bar{\sigma}_{11} h_{02}, \\
& \widehat{\sigma}_{03}=3 \sigma_{11} h_{02}+3 \sigma_{02} \bar{h}_{20}+\sigma_{03}+\frac{3(1+\sqrt{3} i)}{2} \sigma_{02} h_{11} \\
& +\frac{9-3 \sqrt{3} i}{2} h_{02} \bar{h}_{20}+\frac{3(1+\sqrt{3} i)}{2} \bar{\sigma}_{20} h_{02} \text {. }
\end{aligned}
$$

$$
\begin{aligned}
& h_{20}=-\frac{\sigma_{20}}{\sqrt{3} i}, \\
& h_{11}=\frac{2 \sigma_{11}}{3-\sqrt{3} i}, \\
& h_{02}=0,
\end{aligned}
$$

then transformation (40) is determined and map (42) becomes

$$
\phi(n+1)=\lambda_{1} \phi(n)+\frac{\sigma_{02}}{2} \bar{\phi}^{2}(n)+\sum_{i+j=3} \frac{\widehat{\sigma}_{i j}}{i ! j !} \phi^{i}(n) \bar{\phi}^{j}(n)
$$

$$
+O\left(|\phi(n)|^{4}\right)
$$

In order to eliminate some cubic terms, we take the transformation

$$
\begin{aligned}
\phi(n)= & \chi(n)+\frac{1}{6} h_{30} \chi^{3}(n)+\frac{1}{2} h_{21} \chi^{2}(n) \bar{\chi}(n) \\
& +\frac{1}{2} h_{12} \chi(n) \bar{\chi}^{2}(n)+\frac{1}{6} h_{03} \bar{\chi}^{3}(n) .
\end{aligned}
$$
is

$$
\begin{aligned}
\chi(n)= & \phi(n)-\frac{1}{6} h_{30} \phi^{3}(n)-\frac{1}{2} h_{21} \phi^{2}(n) \bar{\phi}(n)-\frac{1}{2} h_{12} \phi(n) \bar{\phi}^{2}(n) \\
& -\frac{1}{6} h_{03} \bar{\phi}^{3}(n)+O\left(|\phi(n)|^{4}\right) .
\end{aligned}
$$

By applying (46) and (47), map (45) becomes

$$
\begin{aligned}
\chi(n+1)= & \lambda_{1} \chi(n)+\frac{\sigma_{02}}{2} \bar{\chi}^{2}(n) \\
& +\sum_{i+j=3} \frac{\tilde{\sigma}_{i j}}{i ! j !} \chi^{i}(n) \bar{\chi}^{j}(n)+O\left(|\chi(n)|^{4}\right),
\end{aligned}
$$

where

$$
\begin{aligned}
& \tilde{\sigma}_{30}=-\frac{3-\sqrt{3} i}{2} h_{30}+\widehat{\sigma}_{30}, \\
& \tilde{\sigma}_{21}=\widehat{\sigma}_{21}, \\
& \widetilde{\sigma}_{12}=\sqrt{3} i h_{12}+\widehat{\sigma}_{12}, \\
& \tilde{\sigma}_{03}=-\frac{3-\sqrt{3} i}{2} h_{03}+\widehat{\sigma}_{03} .
\end{aligned}
$$

Take

Take 


$$
\begin{aligned}
& h_{30}=\frac{2 \widehat{\sigma}_{30}}{3-\sqrt{3} i}, \\
& h_{12}=\frac{\sqrt{3} i}{3} \widehat{\sigma}_{12}, \\
& h_{03}=\frac{2 \widehat{\sigma}_{03}}{3-\sqrt{3} i}, \\
& h_{21}=0,
\end{aligned}
$$

then transformation (46) is determined and map (48) reduces into the following form:

$\chi(n+1)=\lambda_{1} \chi(n)+\frac{\sigma_{02}}{2} \bar{\chi}^{2}(n)+\frac{\widehat{\sigma}_{21}}{2} \chi(n)^{2} \bar{\chi}(n)+O\left(|\chi(n)|^{4}\right)$.

We define $C\left(a_{2}, b_{2}\right):=-(3 / 4)(\sqrt{3} i+1) \sigma_{02}$ and $D\left(a_{2}, b_{2}\right):=-(3 / 4)\left|\sigma_{02}\right|^{2}-(3 / 4)(\sqrt{3} i+1) \widehat{\sigma}_{21}$. From the $1:$ 3 strong resonance bifurcation analysis established in [29], we can get the following theorem.

Theorem 2. Assume that $C\left(a_{2}, b_{2}\right) \neq 0$ and $\operatorname{Re} D\left(a_{2}, b_{2}\right) \neq 0$. Then, model (2) undergoes a $1: 3$ strong resonance bifurcation at $E_{1}^{*}\left(w_{1}^{*}, g_{1}^{*}\right)$. Re $D\left(a_{2}, b_{2}\right) \neq 0$ determines the stability of the bifurcation invariant closed curve. Moreover, model (2) admits a number of the following complex codimension-1 bifurcation curves:

(i) There is a nondegenerate Neimark-Sacker bifurcation at the trivial fixed point $E_{0}$ of (51)

(ii) There is a saddle cycle of periodic three corresponding to saddle fixed points $E_{k}(k=1,2,3)$ of map (51)

(iii) There is a homoclinic structure formed by the stable and unstable invariant manifolds of the period three cycle intersecting transversally in an exponentially narrow parameter region

\section{1 : 4 Strong Resonance}

In this section, we prove that there exists a codimension-2 bifurcation with 1:4 strong resonance of model (2) at $E_{1}^{*}\left(w_{1}^{*}, g_{1}^{*}\right)$. We choose $a$ and $b$ as bifurcation parameters of model (2) and assume that $\left(a_{3}, b_{3}, \mu_{1}, \mu_{2}, \xi_{1}, \xi_{2}\right) \in F_{3}$.

Similar to Section 3, we can also transform $E_{1}^{*}\left(w_{1}^{*}, g_{1}^{*}\right)$ into the origin and then expand the right-hand side of the map into Taylor series. For $\left(a_{3}, b_{3}, \mu_{1}, \mu_{2}, \xi_{1}, \xi_{2}\right) \in F_{3}$, the Jacobian matrix at $E_{1}^{*}\left(w_{1}^{*}, g_{1}^{*}\right)$ is

$$
J\left(E_{1}^{*}\right)=\left(\begin{array}{cc}
1+w_{1}^{*} \alpha_{3} & -w_{1}^{*} \beta_{3} \\
b_{3}-\xi_{2} g_{1}^{*} & 1-\mu_{2}-\xi_{2}\left(N_{1}^{*}+g_{1}^{*}\right)
\end{array}\right),
$$

with

$$
\begin{aligned}
& \alpha_{3}:=\frac{a_{3}\left(1+g_{1}^{*}\right)}{\left(1+N_{1}^{*}\right)^{2}}-\xi_{1}, \\
& \beta_{3}:=\frac{a_{3} w_{1}^{*}}{\left(1+N_{1}^{*}\right)^{2}}+\xi_{1},
\end{aligned}
$$

and it has two complex eigenvalues $\lambda_{1,2}= \pm i$. The corresponding eigenvector $\widetilde{\zeta}_{1} \in \mathbb{C}^{2}$ and adjoint eigenvector $\widetilde{\zeta}_{2} \in \mathbb{C}^{2}$ of $J\left(E_{1}^{*}\right)$ are defined as

$$
\begin{aligned}
& \tilde{\zeta}_{1}=\left(\begin{array}{c}
\tilde{x}_{1} \\
\tilde{y}_{1}
\end{array}\right):=\left(\begin{array}{c}
w_{1}^{*} \beta_{3} \\
1+w_{1}^{*} \alpha_{3}-i
\end{array}\right), \\
& \tilde{\zeta}_{2}=\left(\begin{array}{c}
\tilde{x}_{2} \\
\tilde{y}_{2}
\end{array}\right):=\left(\begin{array}{c}
\frac{1+\left(1+w_{1}^{*} \alpha_{3}\right) i}{2 w_{1}^{*} \beta_{3}} \\
-\frac{1}{2} i
\end{array}\right) .
\end{aligned}
$$

Any vector $Z(n)=(\widehat{x}(n), \widehat{y}(n))^{T} \in \mathbb{R}^{2}$ can be represented as $Z(n)=z(n) \widetilde{\zeta}_{1}+\bar{z}(n) \overline{\widetilde{\zeta}}_{1}$. Thus, for $\left(a_{3}, b_{3}, \mu_{1}\right.$, $\left.\mu_{2}, \xi_{1}, \xi_{2}\right) \in F_{3}$, model (16) can be written in the following complex form:

$$
z(n+1)=\lambda_{1} z(n)+\sum_{2 \leq i+j \leq 3} \frac{\sigma_{i j}}{i ! j !} z^{i}(n) \bar{z}^{j}(n)+O\left(|z(n)|^{4}\right),
$$

where $\lambda_{1}=i$ and $\sigma_{i j}(2 \leq i+j \leq 3)$ are defined as in (39) with $x_{1}, y_{1}, x_{2}, y_{2}, k_{i j}\left(a_{2}, b_{2}\right)$ replaced by $\tilde{x}_{1}, \widetilde{y}_{1}, \widetilde{x}_{2}, \widetilde{y}_{2}, k_{i j}\left(a_{3}, b_{3}\right)$, respectively.

Similar to Section 4, by (40) and (41) we can transform (55) into the following form:

$$
\phi(n+1)=\lambda_{1} \phi(n)+\sum_{2 \leq i+j \leq 3} \frac{\widehat{\sigma}_{i j}}{i ! j !} \phi^{i}(n) \bar{\phi}^{j}(n)+O\left(|\phi(n)|^{4}\right) \text {, }
$$

where

$$
\begin{aligned}
\widehat{\sigma}_{20}= & (i+1) h_{20}+\sigma_{20}, \\
\widehat{\sigma}_{11}= & (i-1) h_{11}+\sigma_{11}, \widehat{\sigma}_{02}=(i+1) h_{02}+\sigma_{02}, \\
\widehat{\sigma}_{30}= & 3(1-i) \sigma_{20} h_{20}+3 \sigma_{11} \bar{h}_{02}+\sigma_{30}-3(i-1) h_{20}^{2}-3(i+1) h_{11} \bar{h}_{02}-3 i \bar{\sigma}_{02} h_{11}, \\
\widehat{\sigma}_{21}= & 2 \sigma_{11} \bar{h}_{11}+(1-2 i) \sigma_{11} h_{20}+(2+i) \sigma_{20} h_{11}+\sigma_{02} \bar{h}_{02}+\sigma_{21}+(3 i+1) h_{20} h_{11} \\
& +2(i-1)\left|h_{11}\right|^{2}-2 i h_{11} \bar{\sigma}_{11}+(i+1)\left|h_{02}\right|^{2}+i \bar{\sigma}_{02} h_{02},
\end{aligned}
$$




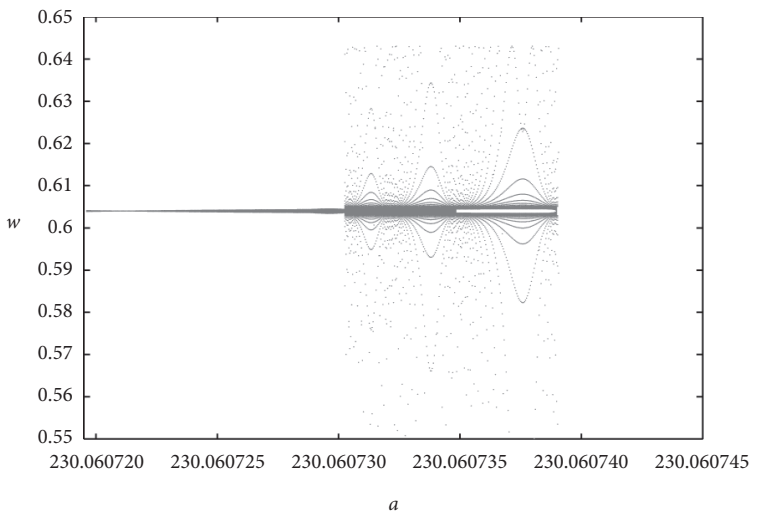

(a)

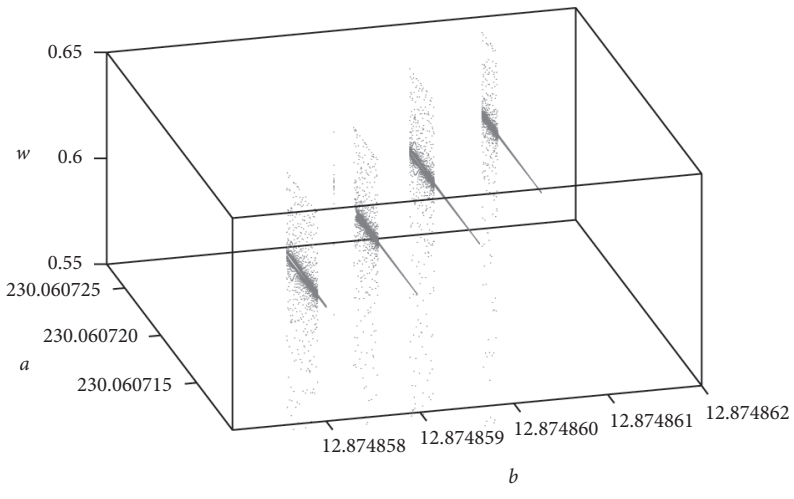

(b)

FIGURE $1: 1: 2$ strong resonance bifurcation diagram at $(0.603987,0.535162)$ : (a) in $(a-w)$ plane with $b=12.87486$ and $(\mathrm{b})$ in $(b-a-w)$ space.

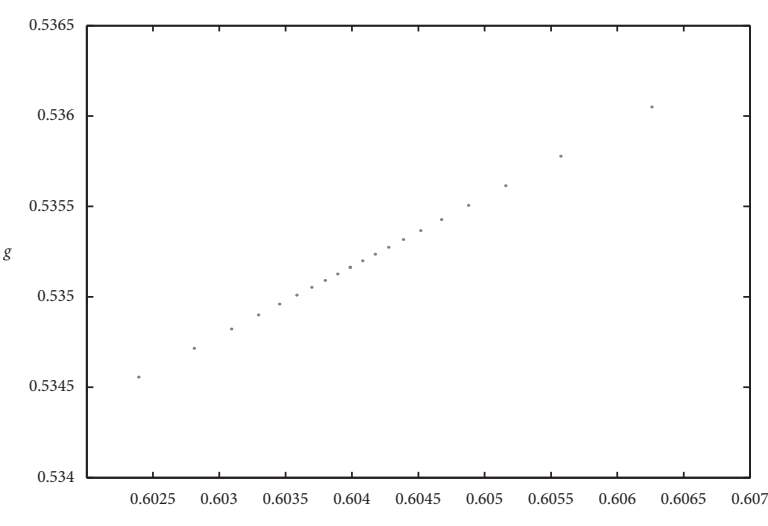

(a)

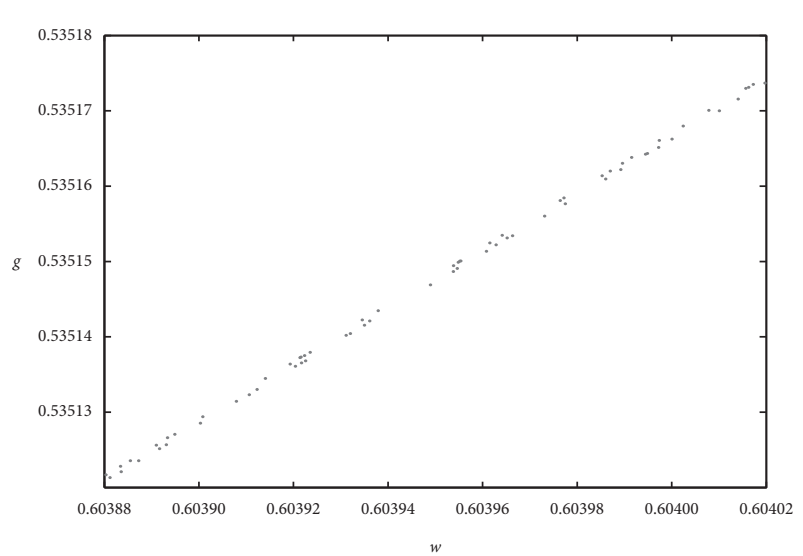

(c)

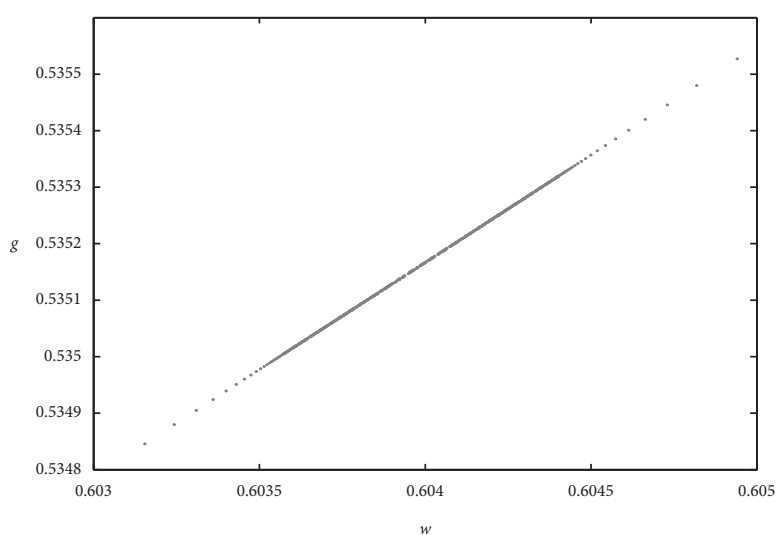

(b)

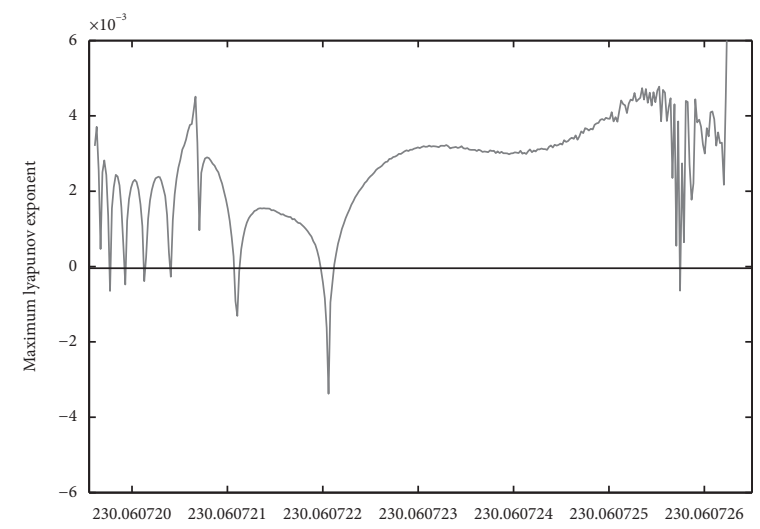

(d)

Figure 2: (a) Phase portraits for $a=230.060692$ and $b=12.87486$. (b) Phase portraits for $a=230.060731$ and $b=12.87486$. (c) Local amplification corresponding to (b). (d) The maximum Lyapunov exponents corresponding to (a). 


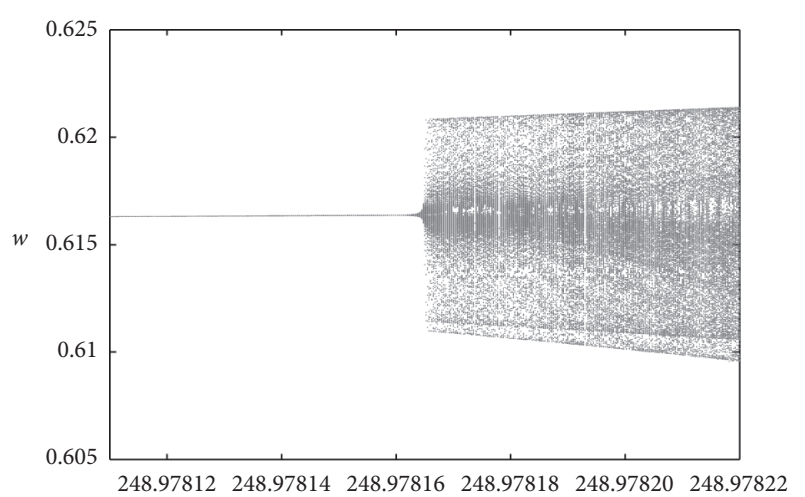

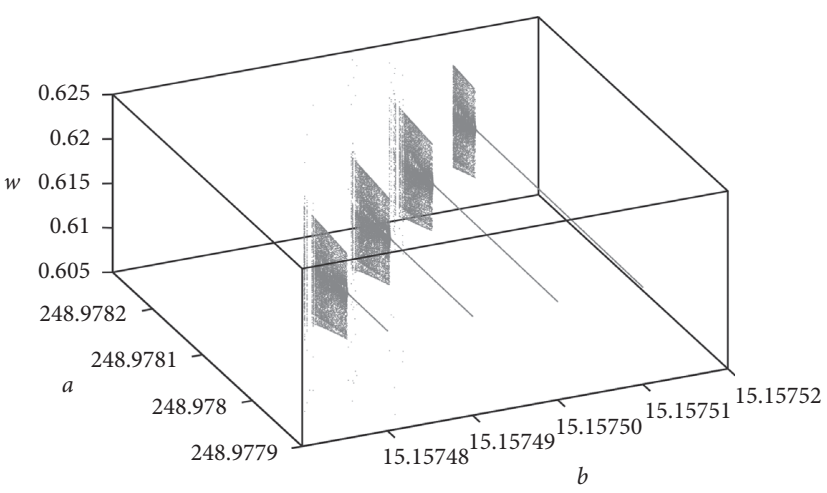

(b)

FIGURE 3: $1: 3$ strong resonance bifurcation diagram at $(0.61636576,0.605198)$ : (a) in $(a-w)$ plane with $b=15.1575017$ and $(b)$ in $(b-a-w)$ space.

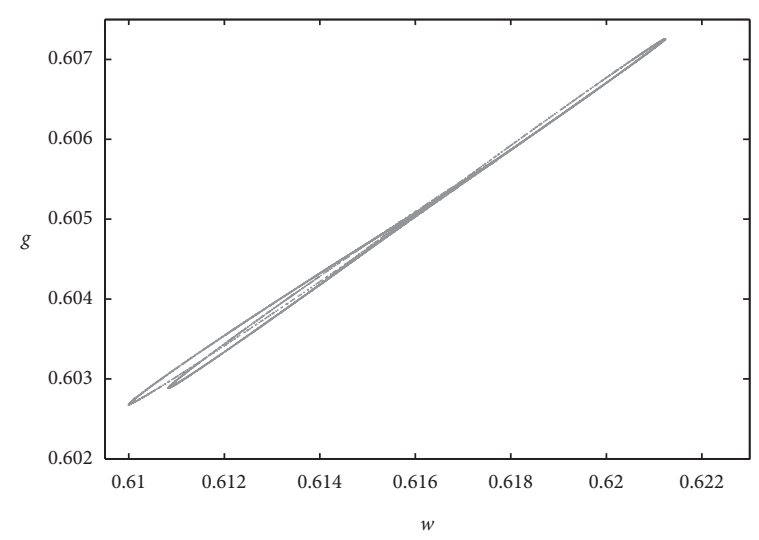

(a)

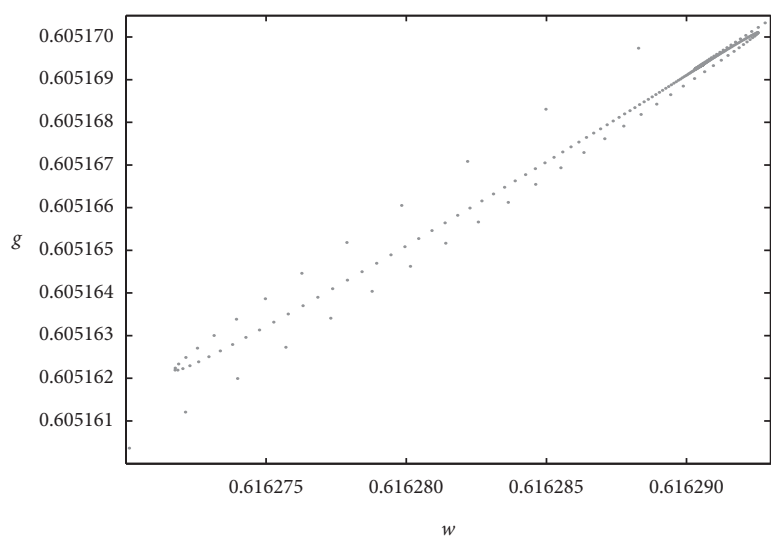

(c)

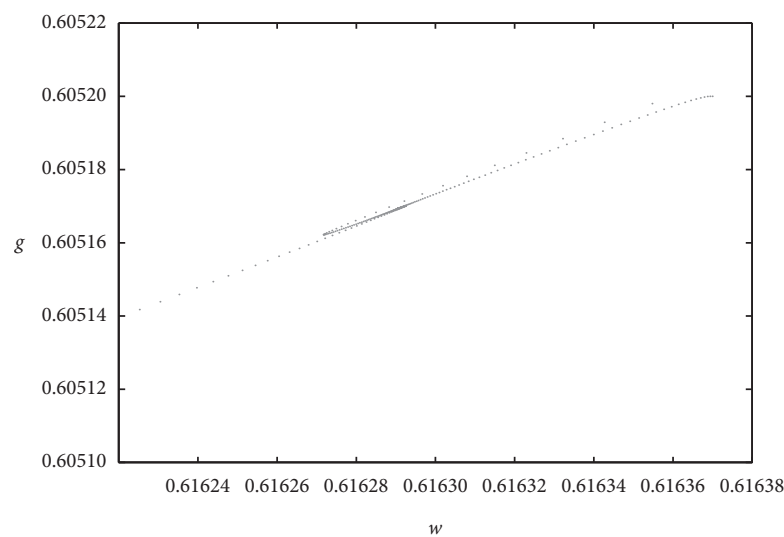

(b)

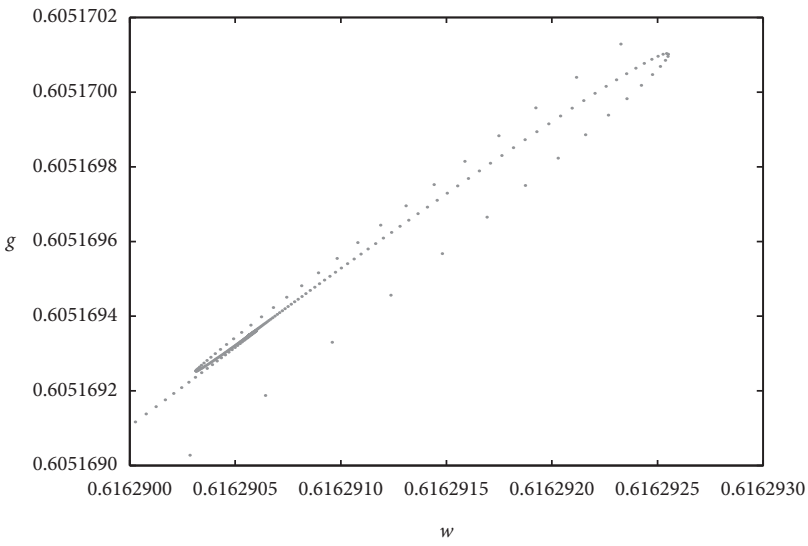

(d)

Figure 4: (a) Phase portraits for $a=248.97819$ and $b=15.1575$. (b) Phase portraits for $a=248.97816$ and $b=15.157509$. (c)-(d) Local amplifications corresponding to (b).

$$
\begin{aligned}
\widehat{\sigma}_{12}= & 2(i+1) \sigma_{11} h_{11}+\sigma_{11} \bar{h}_{20}+2 \sigma_{02} \bar{h}_{11}+\sigma_{20} h_{02}+\sigma_{12}-(i-1) h_{20} h_{02}-2 i \sigma_{02} h_{20} \\
& -2(i+1) h_{11}^{2}-(i+1) h_{11} \bar{h}_{20}-i h_{11} \bar{\sigma}_{20}-2(i-1) h_{02} \bar{h}_{11}+2 i \bar{\sigma}_{11} h_{02}, \\
\widehat{\sigma}_{03}= & 3 \sigma_{11} h_{02}+3 \sigma_{02} \bar{h}_{20}+\sigma_{03}+3(i-1) h_{11} h_{02}+3 i \sigma_{02} h_{11} \\
& +3(i+1) h_{02} \bar{h}_{20}+3 i \bar{\sigma}_{20} h_{02} .
\end{aligned}
$$




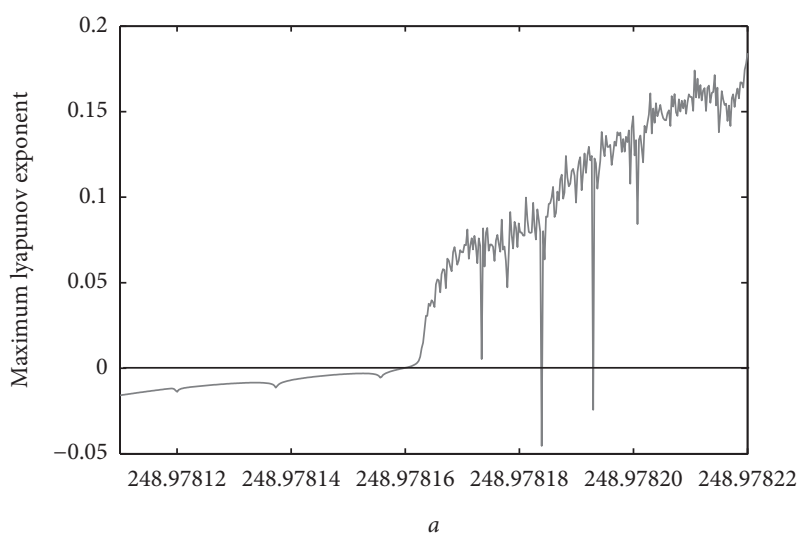

(a)

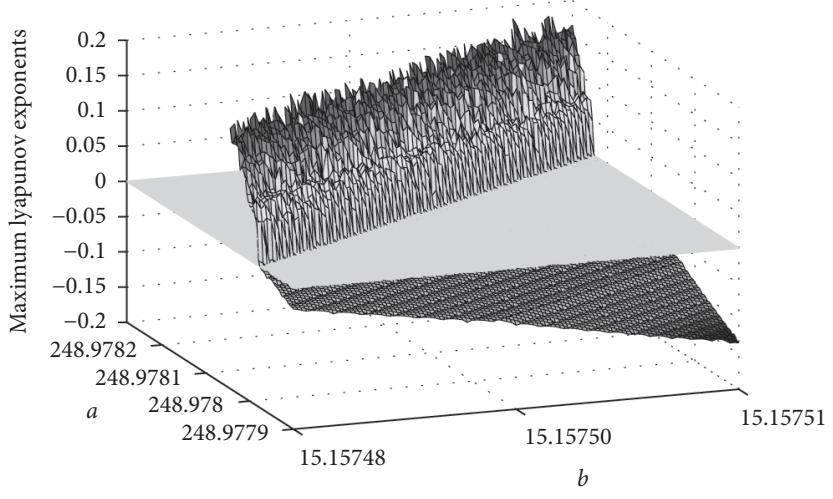

(b)

Figure 5: Maximum Lyapunov exponents of map (2) near $(0.61636576,0.605198)$ as $a$ and $b$ varying: (a) in $(a-$ MLE) plane with $b=$ 15.1575017 and (b) in ( $b-a-$ MLE) space.

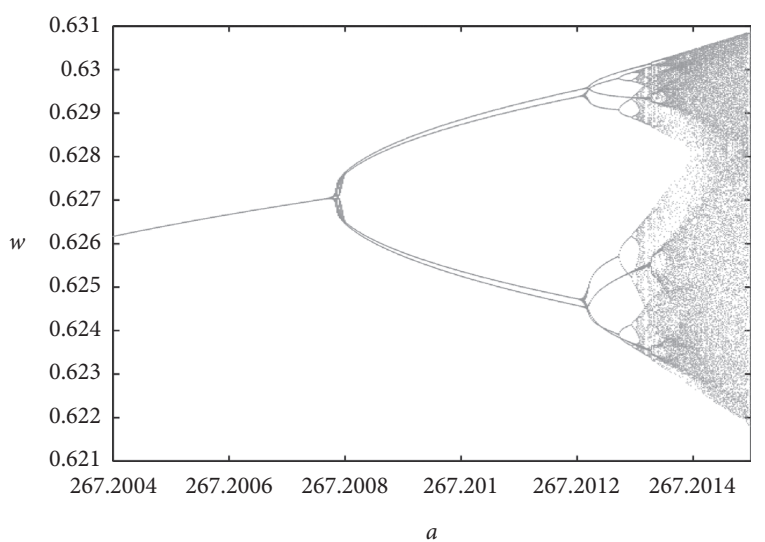

(a)

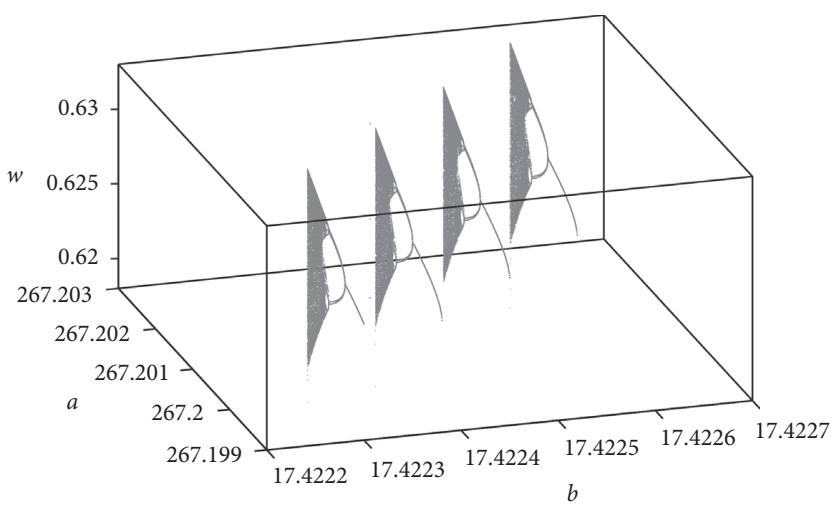

(b)

FIGURE 6: $1: 4$ strong resonance bifurcation diagram at $(0.627048,0.671077)$ : (a) in $(a-w)$ plane with $b=17.42246$ and $(\mathrm{b})$ in $(b-a-w)$ space.

Take

$$
\chi(n+1)=\lambda_{1} \chi(n)+\frac{\widehat{\sigma}_{21}}{2} \chi(n)^{2} \bar{\chi}(n)+\frac{\widehat{\sigma}_{03}}{6} \bar{\chi}^{3}(n)+O\left(|\chi(n)|^{4}\right) .
$$

$$
\begin{aligned}
& h_{20}=-\frac{\sigma_{20}}{1+i}, \\
& h_{11}=\frac{\sigma_{11}}{1-i}, \\
& h_{02}=-\frac{\sigma_{02}}{1+i},
\end{aligned}
$$

then (56) becomes into the following form:

$$
\phi(n+1)=\lambda_{1} \phi(n)+\sum_{i+j=3} \frac{\widehat{\sigma}_{i j}}{i ! j !} \phi^{i}(n) \bar{\phi}^{j}(n)+O\left(|\phi(n)|^{4}\right) .
$$

By (46) and (47), we can transform (59) into the following form as (51):

Let $C\left(a_{3}, b_{3}\right)=-2 i \widehat{\sigma}_{21}$ and $D\left(a_{3}, b_{3}\right)=-(2 / 3) i \widehat{\sigma}_{03}$. If $D\left(a_{3}, b_{3}\right) \neq 0$, we denote $B=\left(\left(C\left(a_{3}, b_{3}\right)\right) /\left(\left|D\left(a_{3}, b_{3}\right)\right|\right)\right)$. From the $1: 4$ strong resonance bifurcation analysis established in [29], we can obtain the following results.

Theorem 3. Assume that $D\left(a_{3}, b_{3}\right) \neq 0, \quad \operatorname{Re} B \neq 0$, and $\operatorname{Im} B \neq 0$. Then, model (2) has a 1:4 strong resonance bifurcation at $E_{1}^{*}\left(w_{1}^{*}, g_{1}^{*}\right)$. B determines the bifurcation scenario near $E_{1}^{*}$. Near the equilibrium $E_{1}^{*}$, there exist twoparameter families of equilibria of order four bifurcation from $E_{1}^{*}$, and one of them is unstable, or attractive or repelling invariant closed curves, which depends on the choices of parameters $a$ and $b$. Moreover, there are many complex 


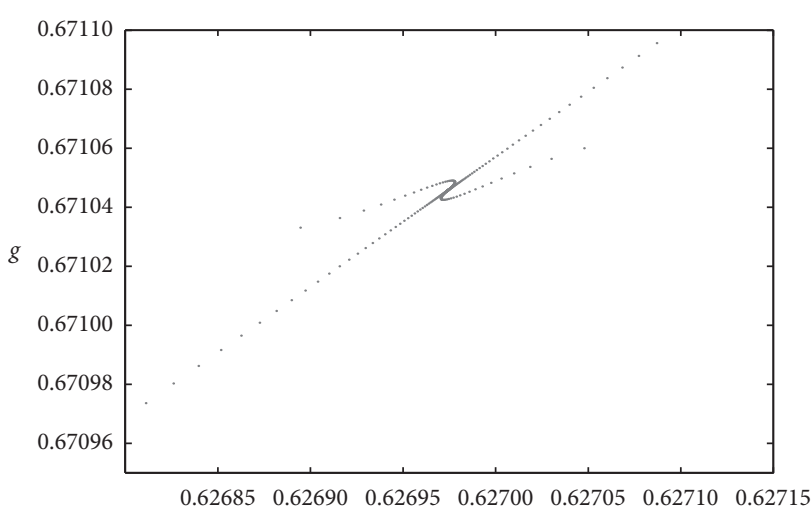

(a)

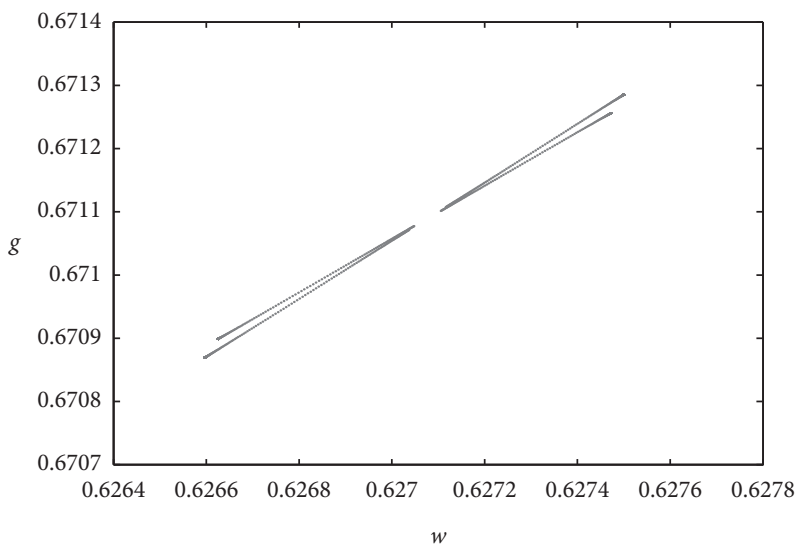

(c)

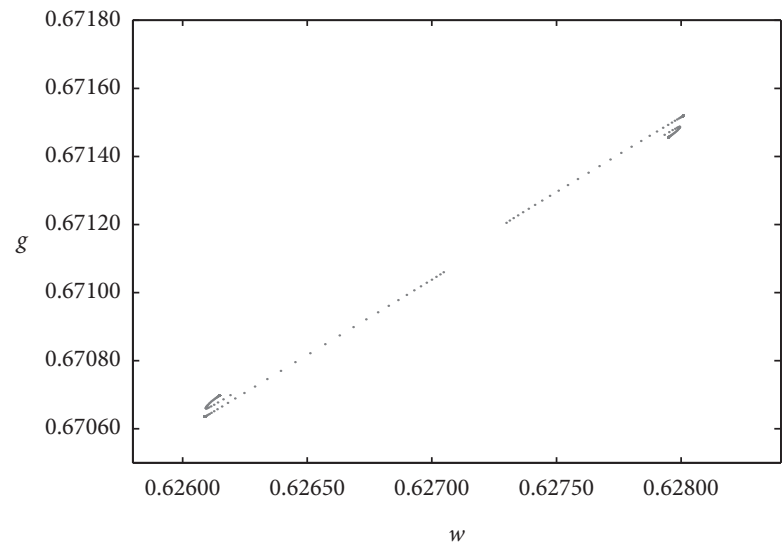

(b)

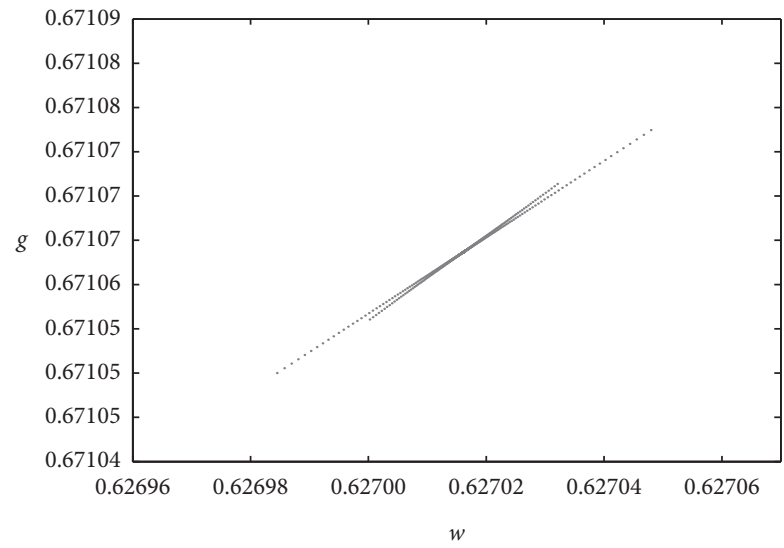

(d)

Figure 7: Phase portraits: (a) $a=267.2007$ and $b=17.422455$; (b) $a=267.2008$ and $b=17.422455$; (c) $a=267.20079$ and $b=17.42246$; (d) $a=267.20076$ and $b=17.42246$.

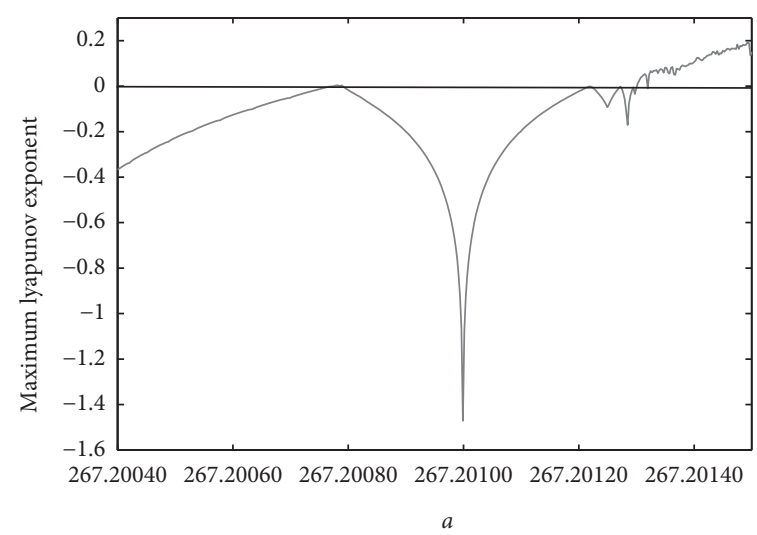

(a)

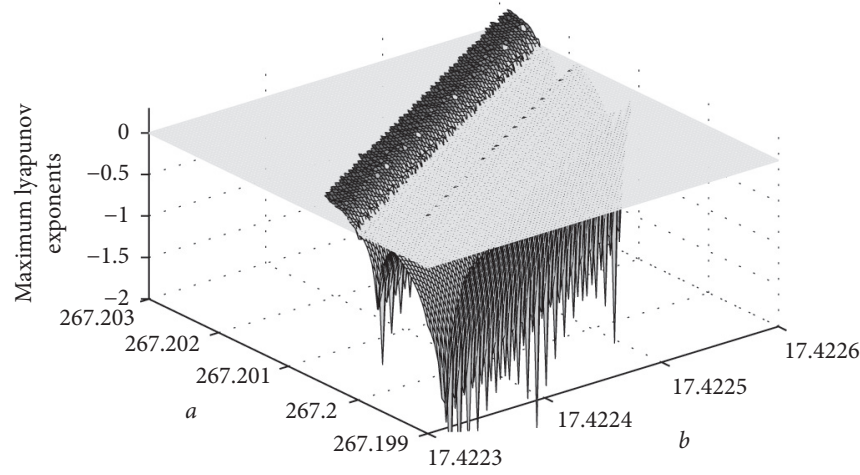

(b)

FIGURE 8: Maximum Lyapunov exponents of map (2) near $(0.627048,0.671077)$ as $a$ and $b$ varying: (a) in $(a-$ MLE) plane with $b=17.42246$ and (b) in ( $b-a-$ MLE) space. 


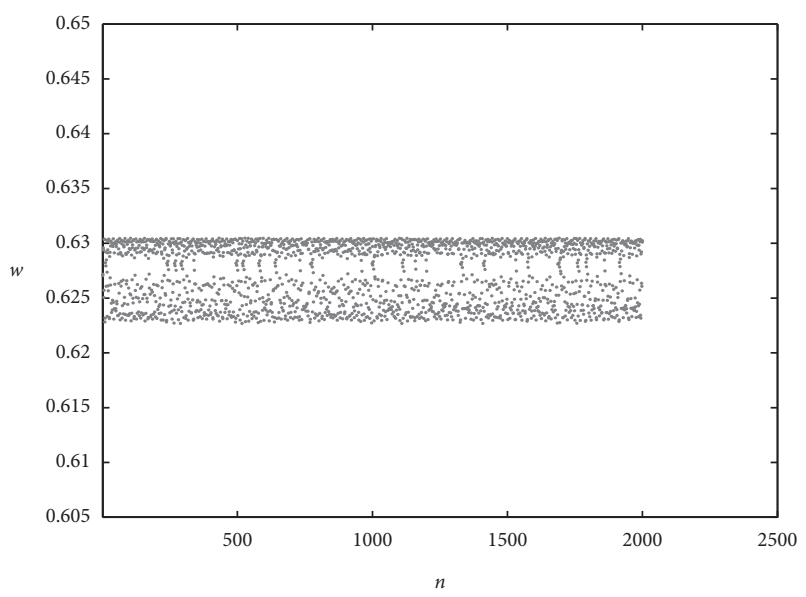

(a)

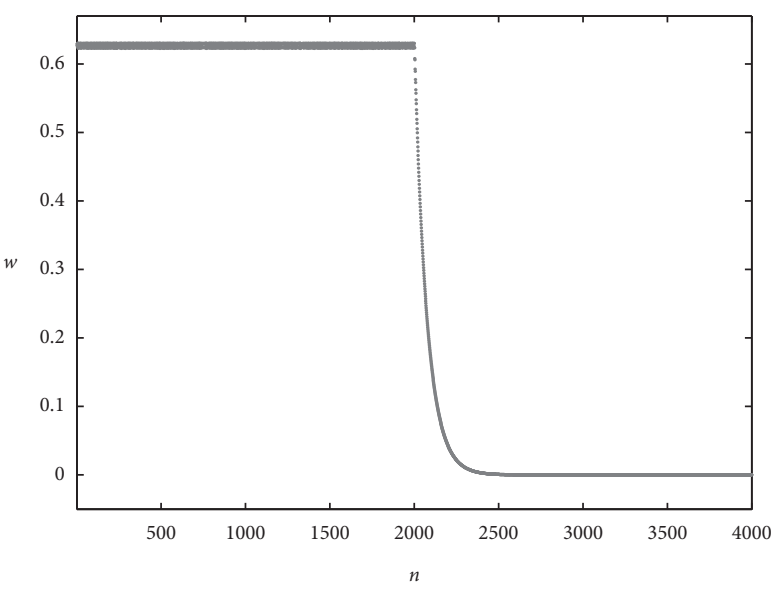

(b)

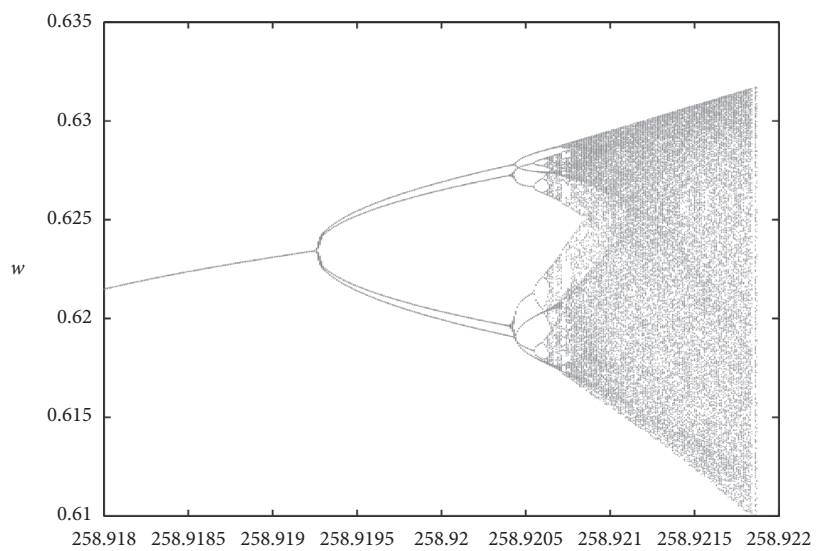

(c)

Figure 9: (a) Chaotic behavior of $w$ for uncontrolled system (2). (b) Time responses for the state $w$ of controlled system (61). (c) Bifurcation diagram of controlled system (68).

codimension-1 bifurcation curves of map (60) in a sufficiently small neighborhood of $\left(a_{3}, b_{3}\right)$.

\section{Numerical Simulations}

In this section, we give a simulation illustration for codimension-2 bifurcations with $1: 2,1: 3$, and $1: 4$ strong resonances. The codimension-2 bifurcation diagrams, maximum Lyapunov exponents, and phase portraits of (2) are presented to illustrate the validity of theoretical results and display the complex dynamical behaviors.

Case 1. Choosing the values of the parameters as $\mu_{1}=8, \mu_{2}=2, \xi_{1}=50$, and $\xi_{2}=11$, we can numerically obtain $a_{1}=230.060722$ and $b_{1}=12.87486$ and equilibrium $E_{1}^{*}\left(w_{1}^{*}, g_{1}^{*}\right)=(0.603987,0.535162)$. The corresponding eigenvalues of $J\left(E_{1}^{*}\right)$ are $\lambda_{1,2}=-1$. By further calculations, we have $C\left(a_{1}, b_{1}\right)=2.1132$ and $D\left(a_{1}, b_{1}\right)=4.2543$. From Theorem 1, map (2) is equivalent to the normal form for $1: 2$ strong resonance bifurcation and $E_{1}^{*}$ is a $1: 2$ strong resonance bifurcation point.
Figure 1(a) shows 2-dimensional bifurcation diagram when $b=b_{1}=12.87486$ and $a$ varies in [230.060715,230.06075], which is a neighborhood of $a_{1}=230.060722$. Figure 1(b) shows 3-dimensional bifurcation diagram when $(a, b)$ vary in a neighborhood of $\left(a_{1}, b_{1}\right)=(230.060722,12.87486)$. Figures 2(a)-2(c) show the phase portraits of (2) near equilibrium $E_{1}^{*}$ for different parameters $a$ and $b$. From the phase portraits, we know that there are periodic orbits and chaos at the corresponding parameter values.

The maximum Lyapunov exponents corresponding to the bifurcation diagram Figure 1(a) are presented in Figure 2(d). From Figure 2(d), we can see that some maximum Lyapunov exponents are positive and some ones are negative, which means that map (2) has chaotic behaviors near the $1: 2$ strong resonance point $E_{1}^{*}$.

Case 2. Choosing the values of the parameters as $\mu_{1}=8, \mu_{2}=2, \xi_{1}=50$, and $\xi_{2}=11$, we can numerically obtain $a_{2}=248.97816$ and $b_{2}=15.1575017$ and equilibrium $E_{1}^{*}\left(w_{1}^{*}, g_{1}^{*}\right)=(0.61636576,0.605198)$. The corresponding 
eigenvalues of $J\left(E_{1}^{*}\right)$ are $\lambda_{1,2}=-(1 / 2) \pm(\sqrt{3} / 2) i$. By further calculations, we have $C\left(a_{2}, b_{2}\right)=1.8958-1.1471 i$ and $D\left(a_{2}, b_{2}\right)=-8.362+5.4278 i$. From Theorem 2, map (2) is equivalent to the normal form for $1: 3$ strong resonance bifurcation and $E_{1}^{*}$ is a $1: 3$ strong resonance bifurcation point.

Figure 3(a) shows 2-dimensional bifurcation diagram when $b=b_{2}=15.1575017$ and $a$ varies in [248.97811,248.97822], which is a neighborhood of $a_{2}=248.97816$. Figure 3(b) shows 3-dimensional bifurcation diagram when $(a, b)$ vary in a neighborhood of $\left(a_{2}, b_{2}\right)=(248.97816,15.1575017)$. Figures 4(a)-4(d) show phase portraits of map (2) near $E_{1}^{*}$ for different $a$ and $b$. From Figure 4(a), we can see that there is a homoclinic structure formed by the stable and unstable invariant manifolds of the period three cycle intersecting transversally in a narrow parameter region.

The maximum Lyapunov exponents corresponding to the bifurcation diagrams Figures 3(a) and 3(b) are presented in Figures 5(a) and 5(b), respectively. We can see that some maximum Lyapunov exponents are positive and some ones are negative, which means that map (2) has chaotic behaviors near the $1: 3$ strong resonance point $E_{1}^{*}$.

Case 3. Choosing the values of the parameters as $\mu_{1}=8, \mu_{2}=2, \xi_{1}=50$, and $\xi_{2}=11$, we can numerically obtain $a_{3}=267.20079$ and $b_{3}=17.42246$ and equilibrium $E_{1}^{*}\left(w_{1}^{*}, g_{1}^{*}\right)=(0.627048,0.671077)$. The corresponding eigenvalues of $J\left(E_{1}^{*}\right)$ are $\lambda_{1,2}= \pm i$. By further calculations, we have $\quad C\left(a_{3}, b_{3}\right)=-1.3731-9.4851 i \quad$ and $D\left(a_{3}, b_{3}\right)=-4.2121+2.2817 i$. From Theorem 3, map (2) is equivalent to the normal form for $1: 4$ strong resonance bifurcation and $E_{1}^{*}$ is a $1: 4$ strong resonance bifurcation point.
Figure 6(a) shows 2-dimensional bifurcation diagram when $b=b_{3}=17.42246$ and $a$ varies in [267.2004, 267.2015], which is a neighborhood of $a_{3}=267.20079$. From Figure 6(a), we can see that there are flip bifurcations occur after Neimark-Sacker bifurcation near the 1:4 strong resonance point. Figure 6(b) shows 3dimensional bifurcation diagram when $(a, b)$ vary in a neighborhood of $\left(a_{3}, b_{3}\right)=(267.20079,17.42246)$. Figures 7(a)-7(d) show phase portraits of map (2) near $E_{1}^{*}$ for different $a$ and $b$. When $a=267.2007$ and $b=17.422455$, the equilibrium is locally stable. When $a$ and $b$ increase, map (2) has chaotic behaviors.

The maximum Lyapunov exponents corresponding to the bifurcation diagrams (Figures 6(a) and 6(b)) are presented in Figures 8(a) and 8(b), respectively. We can see that some maximum Lyapunov exponents are positive and some ones are negative, which means that map (2) has chaotic behaviors near the $1: 4$ strong resonance point $E_{1}^{*}$.

\section{Controller Design}

In this section, we apply two control strategies (tracking control and hybrid control) to (2). By using tracking control, we can annihilate wide mosquitoes and then prevent the transmission of mosquito-borne diseases. By applying hybrid control, we can advance the codimension-2 bifurcation and extend the range of parameter $a$ from the equilibrium to chaos.

7.1. Tracking Control. In this subsection, we design a tracking controller such that the number of wide mosquitoes vanishes gradually as in [23]. Consider a new controlled system

$$
\left\{\begin{array}{l}
w(n+1)=w(n) \exp \left[\frac{a w(n)}{1+w(n)+g(n)}-\left(\mu_{1}+\xi_{1}(w(n)+g(n))\right)\right], \\
g(n+1)=g(n)+b w(n)-\left(\mu_{2}+\xi_{2}(w(n)+g(n))\right) g(n)+R(n),
\end{array}\right.
$$

where $R$ is an exterior control signal. When $R=0$, map (61) has chaos or period orbit under suitable parameter conditions. Let $w^{*}=0$ be our control aim, and we shall design a tracking controller $R$ such that $w(n) \longrightarrow w^{*}=0$ as the number of iterations is sufficiently large.

Theorem 4. If the controller of controlled map (61) is

$$
\begin{aligned}
R(n)= & \frac{-\left(\ln \vartheta(n)+\mu_{1}+\xi_{1}\right)+\sqrt{\left(\ln \vartheta(n)+\mu_{1}+\xi_{1}\right)^{2}+4 \xi_{1}\left(a \Phi_{2}(n)-\ln \vartheta(n)-\mu_{1}\right)}}{2 \xi_{1}} \\
& -\left[\Phi_{2}(n)+g(n)+b w(n)-\left(\mu_{2}+\xi_{2}(w(n)+g(n))\right) g(n)\right],
\end{aligned}
$$


where

$$
\begin{aligned}
\vartheta(n)= & k_{1} \exp \left[-\frac{a w(n)}{1+w(n)+g(n)}+\left(\mu_{1}+\xi_{1}(w(n)\right.\right. \\
& +g(n)))]+k_{2}, \\
\Phi_{2}(n)= & w(n) \exp \left[\frac{a w(n)}{1+w(n)+g(n)}-\left(\mu_{1}+\xi_{1}(w(n)\right.\right. \\
& +g(n)))],
\end{aligned}
$$

and the constants $k_{1}$ and $k_{2}$ are positive constants satisfying all roots of $z^{2}-k_{2} z-k_{1}=0$ lie in the unit circle, then $w(n) \longrightarrow w^{*}=0$ as number of iterations tends to infinity.
Proof. Take the following transformation

$$
\left\{\begin{array}{l}
\Phi_{1}(n)=w(n), \\
\Phi_{2}(n)=w(n) \exp \left[\frac{a w(n)}{1+w(n)+g(n)}-\left(\mu_{1}+\xi_{1}(w(n)+g(n))\right)\right],
\end{array}\right.
$$

then map (61) becomes

$$
\left\{\begin{array}{l}
\Phi_{1}(n+1)=w(n+1)=\Phi_{2}(n), \\
\Phi_{2}(n+1)=\Psi(n),
\end{array}\right.
$$

where

$$
\begin{aligned}
\Psi(n)= & w(n+1) \exp \left[\frac{a w(n+1)}{1+w(n+1)+g(n+1)}-\left(\mu_{1}+\xi_{1}(w(n+1)+g(n+1))\right)\right] \\
= & \Phi_{2}(n) \exp \left\{\frac{a \Phi_{2}(n)}{1+\Phi_{2}(n)+g(n)+b \Phi_{1}(n)-\left(\mu_{2}+\xi_{2}\left(\Phi_{1}(n)+g(n)\right)\right) g(n)+R(n)}\right. \\
& \left.-\left[\mu_{1}+\xi_{1}\left(\Phi_{2}(n)+g(n)+b \Phi_{1}(n)-\left(\mu_{2}+\xi_{2}\left(\Phi_{1}(n)+g(n)\right)\right) g(n)+R(n)\right)\right]\right\} .
\end{aligned}
$$

Taking (62) into (66), we can obtain the following map:

$$
\left\{\begin{array}{l}
\Phi_{1}(n+1)=\Phi_{2}(n) \\
\Phi_{2}(n+1)=k_{1} \Phi_{1}(n)+k_{2} \Phi_{2}(n) .
\end{array}\right.
$$

By choosing suitable positive constants $k_{1}$ and $k_{2}$ such that all roots of $z^{2}-k_{2} z-k_{1}=0$ lie in the unit circle, map (61) after feedback is asymptotically stable. That is, $\Phi_{2}(n) \longrightarrow 0$ as the number of iterations tends to infinity. Therefore, the trajectory of $w$ tracks an ideal state $w^{*}=0$. This completes the proof.

We choose the values of the parameters as $\mu_{1}=8$, $\mu_{2}=2, \xi_{1}=50, \xi_{2}=11, a=267.2014$, and $b=17.42246$, and take $k_{1}=0.75$ and $k_{2}=0.125$ in $R(n)$. When the number of iterations is less than 2000, the chaotic behavior of $w(n)$ for uncontrolled system (2) is presented in Figure 9(a). When $n>2000$, the controller is put on (2). From Figure 9(b), we can see that the trajectory of $w(n)$ tracks ultimately an ideal state $w^{*}=0$.

7.2. Hybrid Control. In this section, we apply hybrid control, which is used in $[28,30]$, to $(2)$. For any $0<\tau<1$, consider a new controlled system

$$
\left\{\begin{array}{l}
w(n+1)=\tau\left\{w(n) \exp \left[\frac{a w(n)}{1+w(n)+g(n)}-\left(\mu_{1}+\xi_{1}(w(n)+g(n))\right)\right]\right\}+(1-\tau) w(n), \\
g(n+1)=\tau\left[g(n)+b w(n)-\left(\mu_{2}+\xi_{2}(w(n)+g(n))\right) g(n)\right]+(1-\tau) g(n),
\end{array}\right.
$$


which has the same equilibria with (2). The Jacobian matrix at equilibrium $E(w, g)$ is

$$
J(E)=\left(\begin{array}{cc}
1+\tau w\left(\frac{a(1+g)}{(1+N)^{2}}-\xi_{1}\right) & -\tau w\left(\frac{a w}{(1+N)^{2}}+\xi_{1}\right) \\
\tau\left(b-\xi_{2} g\right) & 1-\tau \mu_{2}-\tau \xi_{2}(N+g)
\end{array}\right),
$$

its trace is $\operatorname{Tr}(J(E))=2+\tau w\left(\left(\left(a(1+g) /(1+N)^{2}\right)\right)-\xi_{1}\right)-$ $\mu_{2}-\xi_{2}(N+g)=2+(\tau / a) A(N)$, and determinant is $\operatorname{Det}(J(E))=1-(w /(1+N)) \tau^{2} F^{\prime}(N)+(\tau / a) A(N)$. By the analysis in Section 2, we know that only $E_{1}^{*}\left(w_{1}^{*}, g_{1}^{*}\right)$ may be a $1: 2,1: 3$, or $1: 4$ strong resonance point.

Here, we only consider the codimension-2 bifurcation with $1: 4$ strong resonance. Taking $\mu_{1}=8, \mu_{2}=$ $2, \xi_{1}=50, \xi_{2}=11$, and $\tau=0.8$ in (68), we can numerically obtain $a_{4}=258.9192$ and $b_{4}=16.3854$ and equilibrium $E_{1}^{*}\left(w_{1}^{*}, g_{1}^{*}\right)=(0.6234,0.64176)$, which is a $1: 4$ strong resonance point. The bifurcation diagram of controlled system (68) for fixed $b=b_{4}=16.3854$ is presented in Figure $9(\mathrm{c})$ and is very similar to Figure $6(\mathrm{a})$ of the uncontrolled system. Recall that for the uncontrolled system, we have $a_{3}=267.20079$ and $b_{3}=17.42246$. Thus, by using hybrid control, we advance the corresponding codimension-2 bifurcation with $1: 4$ strong resonance. Compared with these two figures, we can also find the range of parameter $a$ from $1: 4$ strong resonance point to chaos in Figure 9(c) is about three times larger than that of Figure 6(a).

\section{Discussion}

In this paper, we analyzed codimension-two bifurcations with $1: 2,1: 3$, and $1: 4$ strong resonances of a discrete wild and sterile mosquito model, which is established from continuous case (1) proposed in [7] by the Euler forward difference method. In $[10,11]$, we have known that continuous model (1) undergoes a sequence of bifurcations such as saddle-node bifurcation, supercritical and subcritical Hopf bifurcation, homoclinic bifurcation, and Bogdanov-Takens bifurcation. Through the theoretical analysis and numerical simulations given in this paper, we find that corresponding discrete mosquito model (2) also has many complex dynamical behaviors, including codimension-1, codimension-2 bifurcations, and chaos. From the impact of the mating rate $a$ and the release rate coefficient $b$ on the dynamics of wide mosquitoes, we know that the release strategy plays an important role in controlling the number of wide mosquitoes. Moreover, two control strategies are applied to model (2). However, whether there is codimension2 bifurcation with $1: 1$ strong resonance is an interesting problem worth considering.

\section{Data Availability}

All data generated or analyzed during this study are included in this article.

\section{Conflicts of Interest}

The authors declare that there are no conflicts of interest regarding the publication of this paper.

\section{Acknowledgments}

This work was supported by the National Natural Science Foundation of China (grant nos. 11801432, 11771373, and 11801429), China Postdoctoral Science Foundation (grant no. 2019M663610), Natural Science Basic Research Plan in Shaanxi Province of China (grant nos. 2018JM1011 and 2019JQ-136), and the Doctoral Scientific Research Foundation of Xi'an Polytechnic University.

\section{References}

[1] H. J. Barclay, "Pest population stability under sterile releases," Researches on Population Ecology, vol. 24, no. 2, pp. 405-416, 1982.

[2] H. Barclay, "Mathematical models for the use of sterile insects," in Sterile Insect Technique, pp. 147-174, Springer Netherlands, Dordrecht, Netherlands, 2005.

[3] Y. Dumont and J. M. Tchuenche, "Mathematical studies on the sterile insect technique for the chikungunya disease and Aedes albopictus," Journal of Mathematical Biology, vol. 65, no. 5, pp. 809-854, 2012.

[4] L. Esteva and H. Mo Yang, "Mathematical model to assess the control of Aedes aegypti mosquitoes by the sterile insect technique," Mathematical Biosciences, vol. 198, no. 2, pp. 132-147, 2005.

[5] J. C. Flores, "A mathematical model for wild and sterile species in competition: immigration," Physica A: Statistical Mechanics and Its Applications, vol. 328, no. 1-2, pp. 214-224, 2003.

[6] J. Li, "Simple mathematical models for interacting wild and transgenic mosquito populations," Mathematical Biosciences, vol. 189, no. 1, pp. 39-59, 2004.

[7] L. Cai, S. Ai, and J. Li, "Dynamics of mosquitoes populations with different strategies for releasing sterile mosquitoes," SIAM Journal on Applied Mathematics, vol. 74, no. 6, pp. 1786-1809, 2014.

[8] W. Jiang, X. Li, and X. Zou, "On a reaction-diffusion model for sterile insect release method on a bounded domain," International Journal of Biomathematics, vol. 7, no. 3, Article ID 1450030, 2014.

[9] J. Li and Z. Yuan, "Modelling releases of sterile mosquitoes with different strategies," Journal of Biological Dynamics, vol. 9, no. 1, pp. 1-14, 2015.

[10] L. Cai, J. Huang, J. Huang, and X. Song, "Bifurcation analysis of a mosquito population model for proportional releasing sterile mosquitoes," Discrete \& Continuous Dynamical Systems-B, vol. 24, no. 11, pp. 6279-6295, 2019.

[11] X. Zhang, J. Shi, and J. Shi, "Bifurcation analysis of a wild and sterile mosquito model," Mathematical Biosciences and Engineering, vol. 16, no. 5, pp. 3215-3234, 2019.

[12] J. Zhang, S. Ruan, P. Yu, and Y. Zhang, "Bifurcation analysis of a mosquito population model with a saturated release rate of sterile mosquitoes," SIAM Journal on Applied Dynamical Systems, vol. 18, no. 2, pp. 939-972, 2019.

[13] H. I. Freedman, Deterministic Mathematical Models in Population Ecology, Monographs and Textbooks in Pure and 
Applied Mathematics, Vol. 57, Marcel Dekker, New York, NY, USA, 1980.

[14] B. S. Goh, Management and Analysis of Biological Populations, Elsevier, Amsterdam, The Netherlands, 1980.

[15] J. D. Murray, Mathematical Biology, Springer, Berlin, Germany, 2nd edition, 1993.

[16] Z. He and X. Jiang, "Bifurcation and chaotic behaviour of a discrete-time variable-territory predator-prey model," IMA Journal of Applied Mathematics, vol. 76, no. 6, pp. 899-918, 2011.

[17] Z. Hu, Z. Teng, and L. Zhang, "Stability and bifurcation analysis of a discrete predator-prey model with nonmonotonic functional response," Nonlinear Analysis: Real World Applications, vol. 12, no. 4, pp. 2356-2377, 2011.

[18] Z. Jing, Y. Chang, and B. Guo, "Bifurcation and chaos in discrete FitzHugh-Nagumo system," Chaos, Solitons \& Fractals, vol. 21, no. 3, pp. 701-720, 2004.

[19] X. Liu and D. Xiao, "Complex dynamic behaviors of a discrete-time predator-prey system," Chaos, Solitons \& Fractals, vol. 32, no. 1, pp. 80-94, 2007.

[20] Q. Chen, Z. Teng, and Z. Hu, "Bifurcation and control for a discrete-time prey-predator model with Holling-IV functional response," International Journal of Applied Mathematics and Computer Science, vol. 23, no. 2, pp. 247-261, 2013.

[21] Q. Din, "Neimark-Sacker bifurcation and chaos control in Hassell-Varley model," Journal of Difference Equations and Applications, vol. 23, no. 4, pp. 741-762, 2017.

[22] E. Kaslik and S. Balint, "Bifurcation analysis for a two-dimensional delayed discrete-time Hopfield neural network," Chaos, Solitons \& Fractals, vol. 34, no. 4, pp. 1245-1253, 2007.

[23] N. Yi, Q. Zhang, P. Liu, and Y. Lin, "Codimension-two bifurcations analysis and tracking control on a discrete epidemic model," Journal of Systems Science and Complexity, vol. 24, no. 6, pp. 1033-1056, 2011.

[24] Q. Chen, Z. Teng, L. Wang, and H. Jiang, "The existence of codimension-two bifurcation in a discrete SIS epidemic model with standard incidence," Nonlinear Dynamics, vol. 71, no. 1-2, pp. 55-73, 2013.

[25] B. Li and Z. He, "1:2 and 1:4 resonances in a two-dimensional discrete Hindmarsh-Rose model," Nonlinear Dynamics, vol. 79, no. 1, pp. 705-720, 2015.

[26] B. Li and Z. He, "1:3 resonance and chaos in a discrete Hindmarsh-Rose model," Journal of Applied Mathematics, vol. 2014, Article ID 896478, 10 pages, 2014.

[27] J. Huang, S. Liu, S. Ruan, and D. Xiao, "Bifurcations in a discrete predator-prey model with nonmonotonic functional response," Journal of Mathematical Analysis and Applications, vol. 464, no. 1, pp. 201-230, 2018.

[28] J. Ren and L. Yu, "Codimension-two bifurcation, chaos and control in a discrete-time information diffusion model," Journal of Nonlinear Science, vol. 26, no. 6, pp. 1895-1931, 2016.

[29] Y. Kuznetsov, Elements of Applied Bifurcation Theory, Springer Verlag, Berlin, Germany, 1998.

[30] X. Luo, G. Chen, B. Wang, and J. Fang, "Hybrid control of period-doubling bifurcation and chaos in discrete nonlinear dynamical systems," Chaos, Solitons \& Fractals, vol. 18, no. 4, pp. 775-783, 2003. 